\title{
HEAVY ELEMENT ABUNDANCES IN GIANT STARS OF THE GLOBULAR CLUSTERS M4 AND M5 ${ }^{1}$
}

\author{
David Yong and Amanda I. Karakas \\ Research School of Astronomy and Astrophysics, Australian National University, Mount Stromlo Observatory, \\ Cotter Road, Weston Creek, ACT 2611, Australia; yong@mso.anu.edu.au, akarakas@mso.anu.edu.au \\ DAVID L. LAMBERT \\ W. J. McDonald Observatory, University of Texas, Austin, TX 78712; d1l@astro.as.utexas.edu \\ Alessandro ChIEFFI \\ Istituto Nazionale di Astrofisica, Istituto di Astrofisica Spaziale e Fisica Cosmica, via Fosso del Cavaliere, I-00133, Rome, Italy; \\ alessandro.chieffi@iasf-roma.inaf.it \\ AND \\ Marco Limongi \\ Istituto Nazionale di Astrofisica-Osservatorio Astronomico di Roma, via Frascati 33, I-00040, Rome, Italy; marco@oa-roma.inaf.it \\ Received 2008 June 26; accepted 2008 August 14
}

\begin{abstract}
We present a comprehensive abundance analysis of 27 heavy elements in bright giant stars of the globular clusters M4 and M5 based on high-resolution, high signal-to-noise ratio spectra obtained with the Magellan Clay Telescope. We confirm and expand on previous results for these clusters by showing that (1) all elements heavier than, and including, Si have constant abundances within each cluster, (2) the elements from $\mathrm{Ca}$ to $\mathrm{Ni}$ have indistinguishable compositions in $\mathrm{M} 4$ and $\mathrm{M} 5$, (3) $\mathrm{Si}, \mathrm{Cu}, \mathrm{Zn}$, and all $s$-process elements are approximately 0.3 dex overabundant in M4 relative to M5, and (4) the $r$-process elements $\mathrm{Sm}, \mathrm{Eu}, \mathrm{Gd}$, and Th are slightly overabundant in $\mathrm{M} 5$ relative to $\mathrm{M} 4$. The cluster-to-cluster abundance differences for $\mathrm{Cu}$ and $\mathrm{Zn}$ are intriguing, especially in light of their uncertain nucleosynthetic origins. We confirm that stars other than Type Ia supernovae must produce significant amounts of $\mathrm{Cu}$ and $\mathrm{Zn}$ at or below the clusters' metallicities. If intermediate-mass AGB stars or massive stars are responsible for the $\mathrm{Cu}$ and $\mathrm{Zn}$ enhancements in $\mathrm{M} 4$, the similar [ $\mathrm{Rb} / \mathrm{Zr}]$ ratios and (preliminary) Mg isotope ratios in both clusters may be problematic for either scenario. For the elements from Ba to $\mathrm{Hf}$, we assume that the $s$ - and $r$-process contributions are scaled versions of the solar $s$ - and $r$-process abundances. We quantify the relative fractions of $s$ - and $r$-process material for each cluster and show that they provide an excellent fit to the observed abundances.
\end{abstract}

Subject headings: Galaxy: abundances — globular clusters: individual (M4, M5) — stars: abundances

Online material: color figures, machine-readable table

\section{INTRODUCTION}

Globular clusters continue to play a vital role in testing many aspects of stellar evolution and stellar nucleosynthesis. Observational and theoretical studies of globular clusters have focused heavily on (1) the star-to-star light element abundance variations (Cottrell \& Da Costa 1981; Langer \& Hoffman 1995; Gratton et al. 2004), (2) the cluster-to-cluster variation in the color distribution of horizontal branch stars, the so-called second-parameter effect (Sandage \& Wildey 1967; Lee et al. 1994; Carretta et al. 2006), and (3) the multiple populations as inferred from large spreads in metallicity and/or detailed structure in color-magnitude diagrams (Butler et al. 1978; Norris \& Da Costa 1995; Bekki \& Norris 2006).

Abundance measurements of the $s$-process and $r$-process elements offer great insight into stellar nucleosynthesis and globular cluster chemical evolution. Aside from M15, a metal-poor cluster that displays a scaled solar $r$-process abundance distribution (Sneden et al. 1997, 2000; Otsuki et al. 2006), in general only a handful of $s$-process elements (e.g., Y, Zr, Ba, La) and the $r$-process element Eu have been measured in globular clusters.

The globular clusters M4 and M5 are particularly well suited for refining our understanding of stellar evolution and stellar nucleo-

\footnotetext{
${ }^{1}$ Based on observations made with the Magellan Clay Telescope at Las Campanas Observatory.
}

synthesis, especially for the neutron-capture elements. Ivans et al. $(1999,2001)$ showed that these clusters have essentially identical metallicities, $[\mathrm{Fe} / \mathrm{H}]=-1.2$, based on high-resolution spectra of large samples, with 36 stars in each cluster. They also showed that the abundance similarities for these clusters extend to numerous $\alpha$ - and Fe-peak elements as well as the $r$-process element Eu. However, the $s$-process elements revealed striking abundance differences between these two clusters. Specifically, the heavy $s$-process elements $\mathrm{Ba}$ and $\mathrm{La}$ are overabundant in $\mathrm{M} 4$ relative to M5 (Ivans et al. 1999, 2001). With the notable exception of $\omega$ Cen, M4 may be uniquely enriched in $s$-process elements among the Galactic globular clusters (Pritzl et al. 2005).

Yong et al. (2008) recently extended the analysis of neutroncapture elements in these two clusters to $\mathrm{Rb}$ and $\mathrm{Pb}, s$-process elements which may be overproduced in metal-poor asymptotic giant branch (AGB) stars (Busso et al. 1999; Travaglio et al. 2001). Whereas $\mathrm{Pb}$ production is dominated by $2-4 M_{\odot}$ lowmetallicity AGB stars (Travaglio et al. 2001), the intermediatemass 4-8 $M_{\odot}$ AGB stars are predicted to dominate $\mathrm{Rb}$ production (van Raai et al. 2008). Not surprisingly, M4 again had higher abundance ratios $[\mathrm{Rb} / \mathrm{Fe}]$ and $[\mathrm{Pb} / \mathrm{Fe}]$ than $\mathrm{M} 5$. However, the abundance ratios $[\mathrm{Rb} / \mathrm{X}]$ for $\mathrm{X}=\mathrm{Y}, \mathrm{Zr}$, and La were very similar in the two clusters indicating that the nature of the $s$-process products is very similar for both clusters but that M4 formed from gas with a higher concentration of these products. A comprehensive 
TABLE 1

Stellar Parameters

\begin{tabular}{|c|c|c|c|c|}
\hline Star & $T_{\text {eff }}$ & $\log g$ & $\xi_{t}$ & {$[\mathrm{Fe} / \mathrm{H}]$} \\
\hline M4 L1411 .................................. & 4025 & 0.80 & 1.75 & -1.23 \\
\hline M4 L1501 ................................ & 4175 & 1.00 & 1.55 & -1.29 \\
\hline 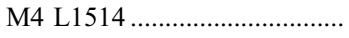 & 3950 & 0.30 & 1.85 & -1.22 \\
\hline M4 L2307 ................................ & 4125 & 0.95 & 1.65 & -1.19 \\
\hline 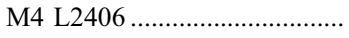 & 4150 & 0.15 & 2.20 & -1.30 \\
\hline 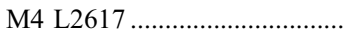 & 4275 & 1.25 & 1.65 & -1.20 \\
\hline M4 L3209 .............................. & 4075 & 0.75 & 1.95 & -1.25 \\
\hline M4 L3413 ….......................... & 4225 & 1.10 & 1.75 & -1.23 \\
\hline 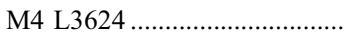 & 4225 & 1.05 & 1.60 & -1.29 \\
\hline M4 L4511 ................................ & 4150 & 1.05 & 1.70 & -1.22 \\
\hline M4 L4611 ............................... & 3925 & 0.15 & 1.45 & -1.09 \\
\hline 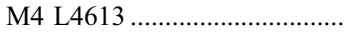 & 3900 & 0.20 & 1.70 & -1.25 \\
\hline M5 IV-81 ................................ & 4050 & 0.30 & 1.90 & -1.28 \\
\hline 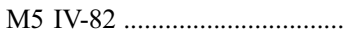 & 4400 & 1.20 & 1.75 & -1.33 \\
\hline
\end{tabular}

study of $s$-process elements in these two clusters promises to provide a novel observational study of the $s$-process at low metallicities as well as valuable clues to the chemical evolution diversity of globular clusters. In this paper we present such an analysis, focusing on a suite of $\alpha$-, Fe-peak, $s$-process, and $r$-process elements.

\section{OBSERVATIONS AND ANALYSIS}

The sample consists of 12 bright giants in M4 and 2 bright giants in M5 observed with the high-resolution spectrograph MIKE (Bernstein et al. 2003) on the Magellan Clay Telescope. These are the same high-quality spectra analyzed by Yong et al. (2008; $R=55,000$, wavelength coverage from 3800 to $8500 \AA$, and $\mathrm{S} / \mathrm{N}=140$ per resolution element at $4000 \AA$ and $\mathrm{S} / \mathrm{N}=800$ per resolution element at $7800 \AA$ ).

The stellar parameters are presented in Table 1 and were determined using a traditional spectroscopic approach. Equivalent widths (EWs) for a set of Fe lines were measured using routines in IRAF. We used the local thermodynamic equilibrium (LTE) stellar line analysis program MOOG (Sneden 1973) and LTE model atmospheres from the Kurucz (1993) grid to derive an abundance for a given line. The effective temperature, $T_{\text {eff }}$, was adjusted until the abundances from $\mathrm{Fe}_{\mathrm{I}}$ lines displayed no trend with the lower excitation potential. The surface gravity, $\log g$, was adjusted until the abundances from $\mathrm{Fe}$ I and $\mathrm{Fe}$ II lines were in agreement. The microturbulent velocity, $\xi_{t}$, was adjusted until there was no trend between the abundances from $\mathrm{Fe}_{\mathrm{I}}$ lines and $\mathrm{EW}$. This process was iterated until self consistent stellar parameters were obtained. Ideally, the trends between abundance and EW and between abundance and lower excitation potential should be exactly zero. Further, the abundances $\log \epsilon\left(\mathrm{Fe}_{\mathrm{I}}\right)$ and $\log \epsilon(\mathrm{Fe}$ II) should be exactly the same. In our analysis, we explored stellar parameters at discrete values. For $T_{\text {eff }}$, we considered values at every $25 \mathrm{~K}$ (e.g., $4000,4025 \mathrm{~K}$, etc.), for $\log g$, we considered values at every 0.05 dex (e.g., 1.00, 1.05 dex, etc.), and for $\xi_{t}$, we considered values at every $0.05 \mathrm{~km} \mathrm{~s}^{-1}$ (e.g., $1.70,1.75 \mathrm{~km} \mathrm{~s}^{-1}$, etc.). We assumed that excitation equilibrium was satisfied when the slope between $\log \epsilon(\mathrm{Fe} \mathrm{I})$ and lower excitation potential was $\leq 0.004$. We assumed that ionization equilibrium was achieved when $\mid \log \epsilon\left(\mathrm{Fe}_{\mathrm{I}}\right)-\log \epsilon(\mathrm{Fe}$ II $) \mid \leq 0.02$ dex. The microturbulent ve-

\footnotetext{
2 IRAF (Image Reduction and Analysis Facility) is distributed by the National Optical Astronomy Observatory, which is operated by the Association of Universities for Research in Astronomy (AURA), Inc., under contract with the National Science Foundation.
}

locity was set when the slope between $\log \epsilon(\mathrm{Fe} \mathrm{I})$ and reduced equivalent width $(\log W / \lambda)$ was $\leq 0.004$. Our stellar parameters are in good agreement with Ivans et al. (1999, 2001). We estimate that the internal errors are $T_{\text {eff }} \pm 50 \mathrm{~K}, \log g \pm 0.2 \mathrm{dex}$, and $\xi_{t} \pm 0.2 \mathrm{~km} \mathrm{~s}^{-1}$. For further details regarding the observations, data reduction, and derivation of stellar parameters, see Yong et al. (2008).

Whenever possible, the abundances for additional elements were determined via EW analysis. For particular lines, the abundances were determined by generating synthetic spectra using MOOG and adjusting the abundance to match the observed spectrum. In Figures 1-4 we show examples of the abundance determination via synthetic spectra. When required, isotopic and/or hyperfine splitting was taken into account. For Pr, we were unable to obtain sufficient information to appropriately treat the hyperfine splitting. The EWs for the 5322.76 А Pr II line range from 24.6 to $65.4 \mathrm{~m} \AA$ and we caution that the abundances may be slightly overestimated. The line list, source of $g f$-values, and EWs are given in Table 2. The adopted solar abundances are given in Table 3. The final abundances are presented in Tables 4 and 5. The abundances for $\mathrm{Rb}, \mathrm{Y}, \mathrm{Zr}, \mathrm{La}, \mathrm{Eu}$, and $\mathrm{Pb}$ were taken from Yong et al. (2008). The abundance dependences on the model parameters are given in Table 6.

For the elements $\mathrm{Si}, \mathrm{Ca}, \mathrm{Sc}, \mathrm{Ti}, \mathrm{V}, \mathrm{Fe}, \mathrm{Ni}, \mathrm{La}$, and $\mathrm{Eu}$, our mean abundances $[\mathrm{X} / \mathrm{Fe}]$ and $[\mathrm{Fe} / \mathrm{H}]$ for M4 and for M5 are in good agreement with the mean cluster values measured by Ivans et al. (1999, 2001) (see Fig. 5). For $\mathrm{Mn}$ and $\mathrm{Cu}$, our mean abundances $[\mathrm{X} / \mathrm{Fe}]$ for M4 and M5 are also in good agreement with the mean cluster values measured by Sobeck et al. (2006) and Simmerer et al. (2003), respectively, who used the same spectra and stellar parameters as Ivans et al. $(1999,2001)$. For all elements previously measured in these clusters, the mean abundance differences are $\Delta[A / B]_{(\text {this study-literature) }}=0.08 \mathrm{dex}(\sigma=0.08 \mathrm{dex})$ and $0.09 \mathrm{dex}$ $(\sigma=0.10$ dex $)$ for M4 and M5, respectively. However, when we consider the mean cluster abundance differences, $\left([A / B]_{\text {this study }}^{\mathrm{M} 4}-\right.$ $\left.[A / B]_{\text {this study }}^{\mathrm{M} 5}\right)-\left([A / B]_{\text {literature }}^{\mathrm{M} 4}-[A / B]_{\text {literature }}^{\mathrm{M} 5}\right)$, our results are in excellent agreement, $-0.01 \mathrm{dex}(\sigma=0.06 \mathrm{dex})$, with Ivans et al. (1999, 2001), Sobeck et al. (2006), and Simmerer et al. (2003). The similarity in the abundance differences, M4 - M5, between the various studies highlights the differential nature of the analyses. That is, the various studies have derived abundances for stars in M4 and M5 that cover a small range of stellar parameters, using homogeneous spectra and analysis techniques. While the similarity in abundance differences is very pleasing, it is not unexpected. We take advantage of the very precise abundance differences to explore the chemical similarities and differences between these two clusters.

\section{RESULTS}

In Figures 6-8, the top panels show the mean abundances $[\mathrm{X} / \mathrm{Fe}]$ and $[\mathrm{Fe} / \mathrm{H}]$ for $\mathrm{M} 4$ and M5 and the bottom panels show the abundance differences, $\Delta[A / B]=[A / B]_{\mathrm{M} 4}-[A / B]_{\mathrm{M} 5}$. The error bars in these figures represent the observed spread $(\sigma)$ in the measured abundances. We note that the observed spread in the measured abundances is in excellent agreement with the predicted spread, $\sigma_{\text {predicted }}-\sigma_{\text {observed (M4) }}=0.00(\sigma=0.05)$ and $\sigma_{\text {predicted }}-$ $\sigma_{\text {observed (M5) }}=0.03(\sigma=0.05)$. For all elements in this study, we therefore regard the abundances as being constant within each cluster. (Note that the $\sigma_{\text {predicted, }}$, taken directly from Table 6 , neglect errors due to EW measurements and continuum placement, which may be small in our high-quality spectra of moderately metal-poor stars. Furthermore, these uncertainties only account for the relative internal uncertainties.) For M4, the light element $\mathrm{Na}$ was found to vary by $\Delta[\mathrm{Na} / \mathrm{Fe}]=0.62 \mathrm{dex}$ within our sample 


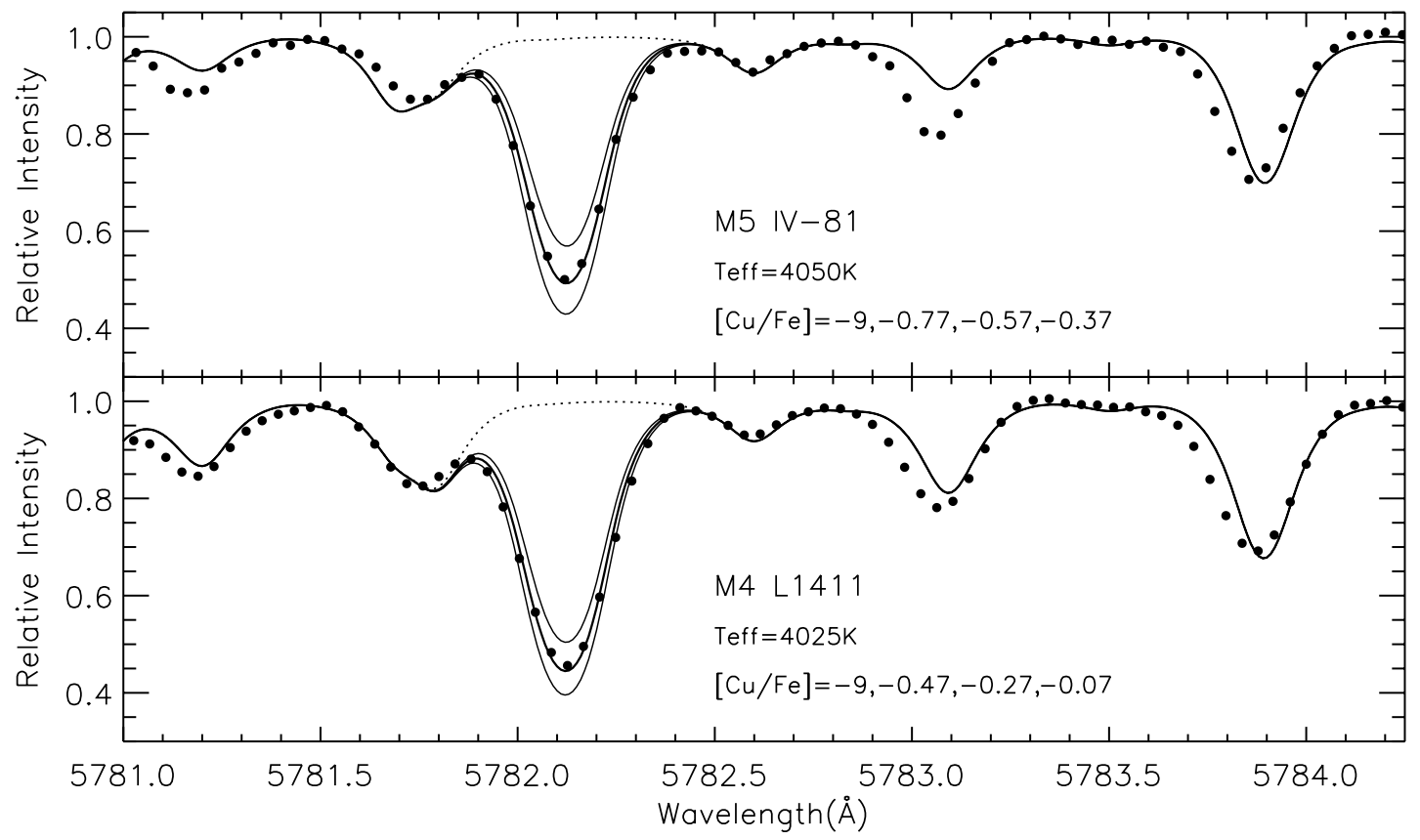

FIG. 1.-Observed spectra ( filled circles) and synthetic spectra (solid and dotted lines) for M5 IV-81 (top) and M4 L1411 (bottom) near the 5782 Å Cu I line. The synthetic spectra show the best fit (thick black line), unsatisfactory fits $\pm 0.2 \mathrm{dex}$ (thin red and blue lines), and a fit with no $\mathrm{Cu}$ (dotted line). [See the electronic edition of the Journal for a color version of this figure.]

(Yong et al. 2008). In this study we find that none of the abundance ratios $[\mathrm{X} / \mathrm{Fe}]$ for $\mathrm{X}=\mathrm{Si}$ to $\mathrm{Th}$ are correlated with $[\mathrm{Na} / \mathrm{Fe}]$.

\subsection{The $\alpha$ - and Fe-Peak Elements, Si to $\mathrm{Zn}$}

In Figure 6 we plot the abundance ratios $[\mathrm{X} / \mathrm{Fe}]$ and $[\mathrm{Fe} / \mathrm{H}]$ for various $\alpha$ - and Fe-peak elements from Si to Zn. We confirm the findings by Ivans et al. $(1999,2001)$ that M4 and M5 have very similar abundances of $\mathrm{Ca}, \mathrm{Sc}, \mathrm{Ti}, \mathrm{V}, \mathrm{Fe}$, and $\mathrm{Ni}$. Our results extend the abundance similarities to the elements $\mathrm{Cr}$ and $\mathrm{Co}$. For every element between $\mathrm{Ca}$ and $\mathrm{Ni}$ inclusive, M4 and M5 have essen- tially identical compositions, $\left\langle\Delta[A / B]_{(\mathrm{M} 4-\mathrm{M} 5)}\right\rangle=0.04(\sigma=0.06)$. The remarkable abundance similarity between M4 and M5 for the elements from $\mathrm{Ca}$ to $\mathrm{Ni}$ is most readily seen in the bottom panel of Figure 6. In Figures 9-11 we plot the spectra for M4 L1411 and M5 IV-81, two stars for which we derived very similar values of $T_{\text {eff }}$. Therefore, any difference between the spectra is most likely due to abundance differences between the two stars. In these figures the similarities in the line strengths of $\mathrm{Ca}, \mathrm{Sc}, \mathrm{Ti}$, $\mathrm{V}, \mathrm{Fe}$, and Co reinforce our findings that these abundances (and the stellar parameters) are very similar in the two stars.

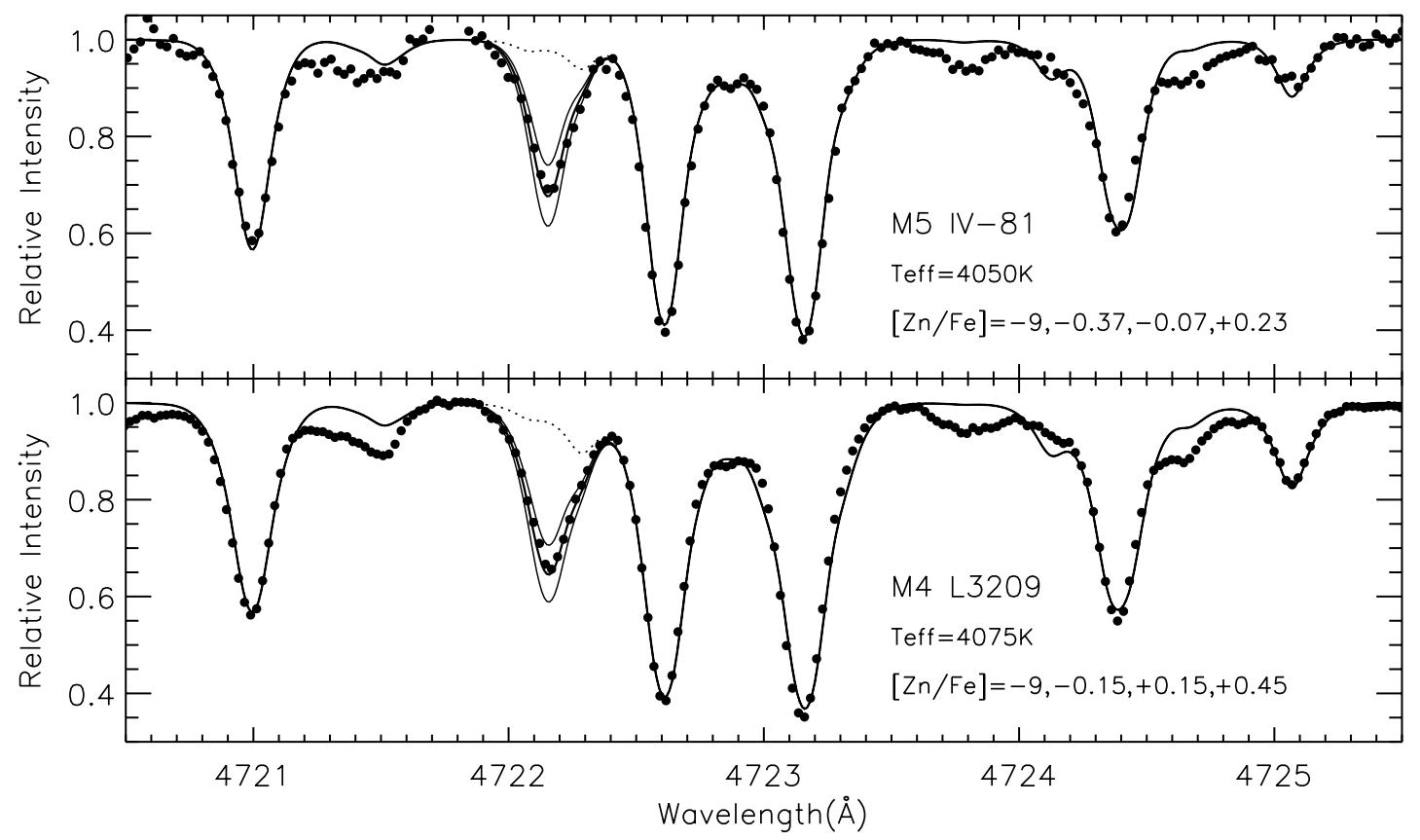

FIG. 2.-Same as Fig. 1, but for the $4722 \mathrm{~A} \mathrm{Zn}$ I line in stars M5 IV-81 and M4 L3209 (unsatisfactory fits \pm 0.3 dex are shown). [See the electronic edition of the Journal for a color version of this figure.] 


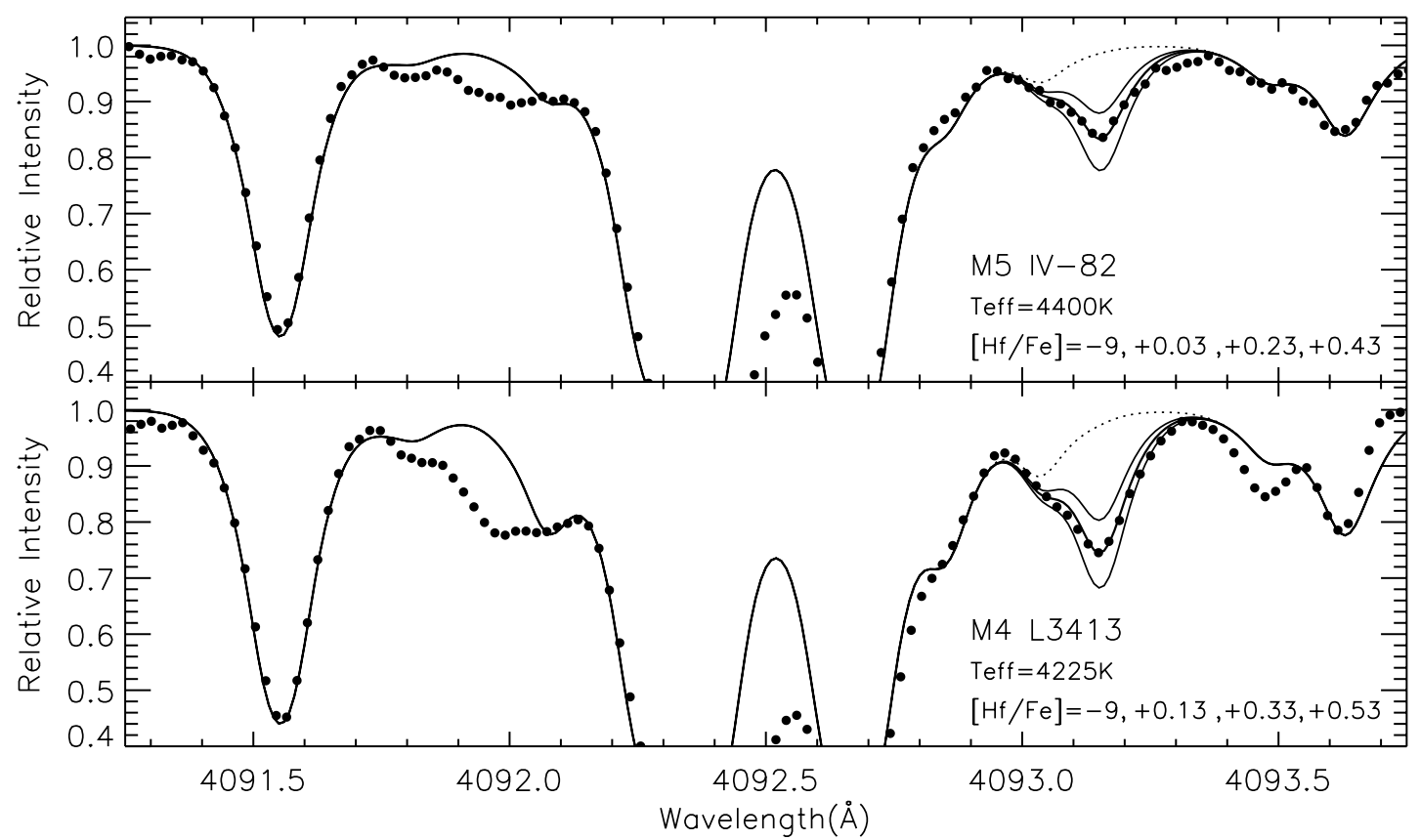

Fig. 3. - Same as Fig. 1, but for the $4093 \AA$ Af II line in stars M5 IV-82 and M4 L3413 (unsatisfactory fits \pm 0.2 dex are shown). [See the electronic edition of the Journal for a color version of this figure.]

The abundance similarities for M4 and M5 do not extend to the elements $\mathrm{Si}, \mathrm{Cu}$, and $\mathrm{Zn}$. For these elements, M4 has [X/Fe] ratios roughly 0.3 dex higher than those in M5.

The case of Si was already documented by Ivans et al. (2001). In Figure 9 lines of Si show considerably different strengths in two stars with very similar values of $T_{\text {eff }}$, which supports the claim that the Si abundances differ between M4 L1411 and M5 IV-81. Curiously, the behavior of Si is very different from the other $\alpha$-elements $\mathrm{Ca}$ and Ti.

The differences in Zn abundances between M4 L3209 and M5 IV-81 can be seen in Figure 2. Although the abundance differ- ences are not immediately obvious when visually comparing line strengths in these two stars, synthetic spectra reveal that M4 L3209 is overabundant in $\mathrm{Zn}$ relative to M5 IV-81.

For the abundances of $\mathrm{Cu}$, differences between M4 and M5 were reported by Simmerer et al. (2003). In Figure 1 the abundance differences between M4 L1411 and M5 IV-81 can be seen from the synthetic spectra. Simmerer et al. (2003) suggested that the $[\mathrm{Cu} / \mathrm{Fe}]$ abundances in both clusters were in agreement with field stars at the same metallicity. (They found a 0.17 dex difference in $[\mathrm{Fe} / \mathrm{H}]$ between these clusters, with $\mathrm{M} 4$ being the more metal-rich cluster.) Inspection of their Figure 6 shows that while

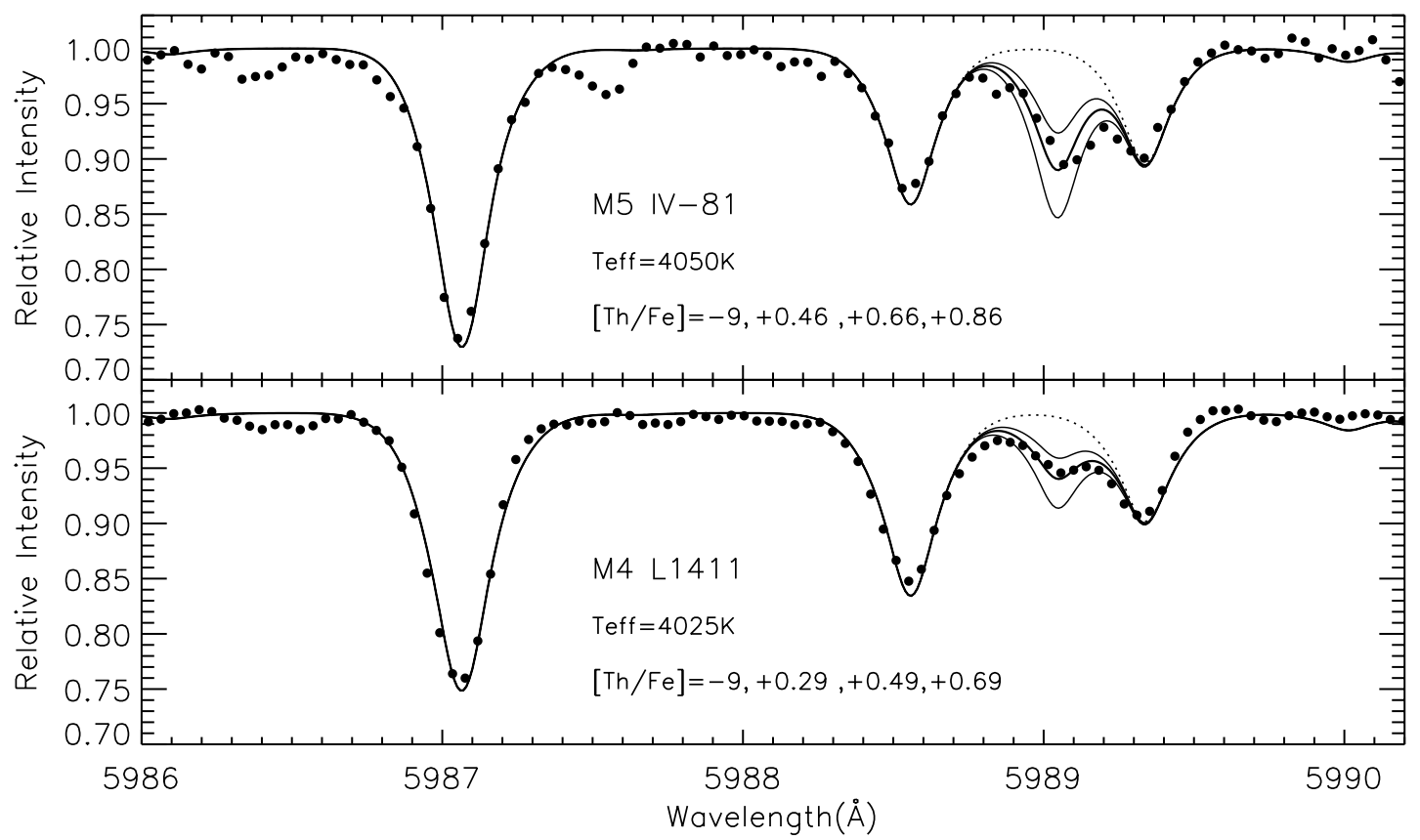

FIG. 4.-Same as Fig. 1, but for the $5989 \AA \AA$ Th II line (unsatisfactory fits \pm 0.2 dex are shown). [See the electronic edition of the Journal for a color version of this figure.] 
TABLE 2

EQuivalent WidThS

\begin{tabular}{cccccc}
\hline \hline $\begin{array}{c}\text { Wavelength } \\
(\AA)\end{array}$ & Species & $\begin{array}{c}\chi \\
(\mathrm{eV})\end{array}$ & $\log g f$ & Source $g f$ & M4 L1411 \\
\hline $5665.554 \ldots \ldots .$. & 14.0 & 4.92 & -2.04 & RC02 & 49.2 \\
$5690.430 \ldots \ldots .$. & 14.0 & 4.93 & -1.83 & RC02 & 48.4 \\
$5948.550 \ldots \ldots .$. & 14.0 & 5.08 & -1.23 & RC02 & 76.3 \\
$6142.490 \ldots \ldots .$. & 14.0 & 5.62 & -1.48 & IK01 & 21.2 \\
$6145.020 \ldots \ldots .$. & 14.0 & 5.61 & -1.37 & RC02 & 29.7 \\
$6155.134 \ldots \ldots .$. & 14.0 & 5.62 & -0.76 & RC02 & 57.0 \\
$6721.840 \ldots \ldots .$. & 14.0 & 5.86 & -0.94 & RC02 & 29.6 \\
\hline
\end{tabular}

NoтE.-Table 2 is published in its entirety in the electronic edition of the Astrophysical Journal. A portion is shown here for guidance regarding its form and content.

ReFERENCES.-CM05: Cohen \& Meléndez 2005; CS02: Cunha et al. 2002; DL03: Den Hartog et al. 2003; DL06: Den Hartog et al. 2006; IK01: Ivans et al. 1999; KB95: Kurucz \& Bell 1995; LD06: Lawler et al. 2006; LH07: Lawler et al. 2007; LUCK: R. E. Luck private communication; ND95: Norris et al. 1995; NZ02: Nilsson et al. 2002; PN00: Prochaska et al. 2000 ; RC02: Ramírez \& Cohen 2002; RT03: Reddy et al. 2003; SS00: Smith et al. 2000.

$[\mathrm{Cu} / \mathrm{Fe}]$ in halo field stars (drawn exclusively from Mishenina et al. 2002) appears to be falling rapidly with decreasing metallicity, there are no field stars in the metallicity regime between M4 and M5. The sparse data set reveals that for the five field halo stars closest in metallicity to M4 and M5, the $[\mathrm{Cu} / \mathrm{Fe}]$ values cover roughly 0.6 dex. However, some of the Mishenina et al. (2002) comparison field halo stars may be regarded as being (slightly) chemically peculiar. HD 6833 has a higher than usual ratio $[\mathrm{Zr} / \mathrm{Fe}]=$ +0.48 (Fulbright 2000) and HD 166161 has a higher than usual ratio $\log \epsilon(\mathrm{La} / \mathrm{Eu})=0.71$ (Simmerer et al. 2004). For these two stars, the $[\mathrm{Cu} / \mathrm{Fe}]$ ratios may not be representative of the general halo trend and therefore, the claim that M4 and M5 have $\mathrm{Cu}$ abundances in agreement with field halo stars needs to be reexamined. Recent Cu measurements by Primas \& Sobeck (2008) more clearly define the $[\mathrm{Cu} / \mathrm{Fe}]$ versus $[\mathrm{Fe} / \mathrm{H}]$ trend in field halo stars. Their results suggest that the 0.25 dex difference in $[\mathrm{Cu} / \mathrm{Fe}]$ between M4 and M5 may exceed the intrinsic spread for the small range in $[\mathrm{Fe} / \mathrm{H}]$ spanned by the two clusters. However, we note that such abundance comparisons between different studies can be problematic due to (unknown) systematic differences.

\subsection{The s-Process and r-Process Elements, $\mathrm{Rb}$ to $\mathrm{Th}$}

In Figure 7 we plot the abundance ratios $[\mathrm{X} / \mathrm{Fe}]$ for various $s$-process and $r$-process elements from $\mathrm{Rb}$ to $\mathrm{Gd}$, and in Figure 8 we plot the abundance ratios for all elements including $\mathrm{Hf}, \mathrm{Pb}$, and Th. Ivans et al. $(1999,2001)$ showed that the $s$-process elements $\mathrm{Ba}$ and La are overabundant in M4 relative to M5, and Yong et al. (2008) extended the abundance differences to the $s$-process elements $\mathrm{Rb}$ and $\mathrm{Pb}$. We find that every $s$-process element shows overabundances in M4 relative to M5. In Figures 9 ( Zr), 10 ( Y and Mo), and 11 ( $\mathrm{Y}, \mathrm{Zr}$, and $\mathrm{Nd}$ ), various $s$-process elements have considerably different line strengths, reinforcing the claim that the abundances differ between M4 L1411 and M5 IV-81. The nine $s$-process elements measured in M4 and M5, Rb, Sr, Y, Zr, Mo, $\mathrm{Ba}, \mathrm{La}, \mathrm{Ce}$, and $\mathrm{Pb}$, have $\left\langle\Delta[\mathrm{X} / \mathrm{Fe}]_{(\mathrm{M} 4-\mathrm{M} 5)}\right\rangle=0.38(\sigma=0.14)$. Although the abundances are derived from a single line in a crowded region, the behavior of $\mathrm{Pb}, \Delta[\mathrm{Pb} / \mathrm{Fe}]_{(\mathrm{M} 4-\mathrm{M} 5)}=0.65$, may differ from that of the other $s$-elements in these clusters. Recent observations of $\mathrm{Pb}$ by Aoki \& Honda (2008) in a sample of field halo stars without large carbon enhancements show that M5 has a $[\mathrm{Pb} / \mathrm{Fe}]$ ratio comparable to field stars at the same metallicity. The
TABLE 3

Adopted Solar Abundances

\begin{tabular}{|c|c|c|}
\hline Species & $\log \epsilon(\mathrm{X})$ & Source \\
\hline $\mathrm{Si}$ & 7.55 & 1 \\
\hline 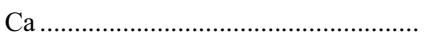 & 6.31 & 2 \\
\hline Sc $\ldots \ldots \ldots \ldots \ldots \ldots \ldots \ldots$ & 3.05 & 2 \\
\hline $\mathrm{Ti} \ldots \ldots \ldots \ldots \ldots \ldots \ldots$ & 4.90 & 2 \\
\hline $\mathrm{V}$ & 4.00 & 2 \\
\hline $\mathrm{Cr}$ & 5.64 & 2 \\
\hline Mn & 5.39 & 2 \\
\hline Fe. & 7.50 & 1 \\
\hline 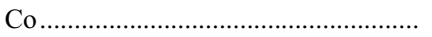 & 4.92 & 2 \\
\hline Ni..................... & 6.23 & 2 \\
\hline $\mathrm{Cu} . . \ldots \ldots \ldots \ldots \ldots$ & 4.21 & 1 \\
\hline $\mathrm{Zn} \ldots \ldots \ldots \ldots \ldots \ldots$ & 4.60 & 1 \\
\hline 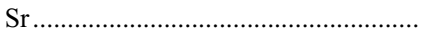 & 2.92 & 1 \\
\hline 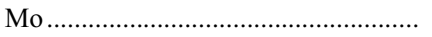 & 1.92 & 1 \\
\hline $\mathrm{Ce}$ & 1.58 & 1 \\
\hline $\operatorname{Pr}$ & 0.58 & 3 \\
\hline $\mathrm{Nd}$ & 1.45 & 3 \\
\hline . & 1.00 & 3 \\
\hline 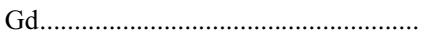 & 1.11 & 3 \\
\hline 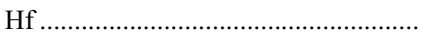 & 0.88 & 3 \\
\hline Th & 0.06 & 3 \\
\hline
\end{tabular}

REFERENCES.-(1) Grevesse \& Sauval 1998; (2) Asplund et al. 2005; (3) Grevesse et al. 2007.

Aoki \& Honda (2008) results also show that the globular clusters NGC 6752 and M13 have $[\mathrm{Pb} / \mathrm{Fe}]$ ratios typical of field halo stars at the same metallicity (Yong et al. 2006b).

The sole $r$-process element measured by Ivans et al. (1999, 2001 ) is Eu, and it was found to have a slightly higher abundance in M5 relative to M4, in contrast to the behavior of the $s$-elements. In this study, we have measured the abundances for three additional $r$-process elements, Sm, Gd, and Th. In Figure 4, we show synthetic spectra fits to the $5989 \AA$ Th II line in which M5 IV-81 has a slightly higher abundance than M4 L1411. The $5989 \AA$ Th II line was also used by Aoki et al. (2007) and Figure 4 shows that in the absence of large Th enhancements, abundance estimates are only possible from extremely high $\mathrm{S} / \mathrm{N}$ spectra. Ivans et al. (2006) measured Th abundances in the $r$-process-rich star HD 221170 and found that the abundances from $5989 \AA$ Th II line agreed with measurements from other Th lines. In Figure 11, the $4642 \AA$ Sm II line has similar strengths in M4 L1411 and M5 IV-81.

In Figure 7 there is a trend in which $\Delta[\mathrm{X} / \mathrm{Fe}]_{(\mathrm{M} 4-\mathrm{M} 5)}$ decreases as the atomic number increases for the $r$-process elements $\mathrm{Sm}$, $\mathrm{Eu}$, and $\mathrm{Gd}$. However, this trend does not extend to the Th abundances (see Fig. 8). Overall, all four $r$-process elements, Sm, Eu, $\mathrm{Gd}$, and Th, are slightly underabundant in M4 relative to M5, $\left\langle\Delta[\mathrm{X} / \mathrm{Fe}]_{(\mathrm{M} 4-\mathrm{M} 5)}\right\rangle=-0.13(\sigma=0.09)$.

For the neutron-capture elements $\mathrm{Pr}, \mathrm{Nd}$, and $\mathrm{Hf}$, the solar abundances may be equally attributed to the $s$-process and $r$-process. Given the behavior of the $s$ - and $r$-elemental abundances in these clusters, we would therefore expect $\mathrm{Pr}, \mathrm{Nd}$, and $\mathrm{Hf}$ to be overabundant in M4 relative to M5, but that the magnitude of the enhancement would lie midway between the bulk of the $s$ - and $r$ - elements. Indeed, the abundance differences $\Delta[\mathrm{Pr} / \mathrm{Fe}]_{(\mathrm{M} 4-\mathrm{M} 5)}=+0.09, \Delta[\mathrm{Nd} / \mathrm{Fe}]_{(\mathrm{M} 4-\mathrm{M} 5)}=+0.12$, and $\Delta[\mathrm{Hf} / \mathrm{Fe}]_{(\mathrm{M} 4-\mathrm{M} 5)}=+0.11$, all lie midway between the heavy $s$-elements, $\Delta\left\langle[\mathrm{Ba}, \mathrm{La}, \mathrm{Ce} / \mathrm{Fe}]_{(\mathrm{M} 4-\mathrm{M} 5)}\right\rangle=+0.36$, and the $r$-process elements $\Delta\langle[\mathrm{Sm}, \mathrm{Eu}, \mathrm{Gd} / \mathrm{Fe}]\rangle_{(\mathrm{M} 4-\mathrm{M} 5)}=-0.12$. For Pr, these results indicate that either hyperfine structure does not significantly 
TABLE 4

Abundance Ratios [ $\mathrm{X} / \mathrm{Fe}$ ] for $\mathrm{Ca}$ to $\mathrm{Zn}$

\begin{tabular}{|c|c|c|c|c|c|c|c|c|c|c|c|}
\hline Star & {$[\mathrm{Si} / \mathrm{Fe}]$} & {$[\mathrm{Ca} / \mathrm{Fe}]$} & {$[\mathrm{Sc} / \mathrm{Fe}]$} & {$[\mathrm{Ti} / \mathrm{Fe}]$} & {$[\mathrm{V} / \mathrm{Fe}]$} & {$[\mathrm{Cr} / \mathrm{Fe}]$} & {$[\mathrm{Mn} / \mathrm{Fe}]$} & {$[\mathrm{Co} / \mathrm{Fe}]$} & {$[\mathrm{Ni} / \mathrm{Fe}]$} & {$[\mathrm{Cu} / \mathrm{Fe}]$} & {$[\mathrm{Zn} / \mathrm{Fe}]$} \\
\hline M4 L1411 ................. & 0.58 & 0.43 & 0.17 & 0.42 & 0.18 & 0.11 & -0.16 & 0.04 & 0.10 & -0.27 & $\ldots$ \\
\hline M4 L1501 ................... & 0.64 & 0.45 & 0.21 & 0.35 & 0.18 & 0.07 & -0.23 & 0.01 & 0.15 & -0.27 & 0.24 \\
\hline M4 L1514 ................. & 0.51 & 0.34 & 0.00 & 0.44 & 0.15 & 0.06 & $\ldots$ & 0.00 & 0.09 & -0.33 & 0.27 \\
\hline M4 L2307 ................. & 0.54 & 0.47 & 0.17 & 0.39 & 0.17 & 0.09 & -0.23 & 0.01 & 0.14 & -0.31 & 0.19 \\
\hline M4 L2406 ................... & 0.46 & 0.19 & 0.04 & 0.16 & 0.04 & -0.12 & -0.36 & -0.03 & 0.06 & -0.25 & 0.30 \\
\hline M4 L2617 ................. & 0.61 & 0.44 & 0.04 & 0.38 & 0.23 & 0.05 & -0.23 & 0.01 & 0.10 & -0.31 & 0.15 \\
\hline M4 L3209 .................. & 0.60 & 0.44 & 0.02 & 0.50 & 0.28 & 0.14 & -0.15 & 0.07 & 0.17 & -0.2 & 0.15 \\
\hline M4 L3413 ................. & 0.44 & 0.42 & 0.13 & 0.41 & 0.20 & 0.11 & -0.24 & 0.01 & 0.05 & -0.22 & 0.13 \\
\hline M4 L3624 .................. & 0.62 & 0.47 & 0.15 & 0.38 & 0.21 & 0.11 & -0.25 & 0.03 & 0.14 & -0.22 & 0.19 \\
\hline M4 L4511 ................. & 0.68 & 0.43 & 0.17 & 0.33 & 0.15 & 0.05 & -0.24 & 0.01 & 0.10 & -0.23 & 0.17 \\
\hline M4 L4611 ................... & 0.70 & 0.50 & 0.20 & 0.59 & 0.25 & 0.13 & $\ldots$ & -0.13 & 0.17 & -0.46 & 0.29 \\
\hline M4 L4613 ................. & 0.52 & 0.42 & 0.10 & 0.55 & 0.25 & 0.16 & $\ldots$ & -0.03 & 0.14 & -0.31 & 0.20 \\
\hline M5 IV-81 ................... & 0.32 & 0.37 & 0.08 & 0.40 & 0.11 & 0.08 & -0.24 & -0.08 & -0.02 & -0.52 & -0.07 \\
\hline M5 IV-82 .................. & 0.32 & 0.37 & 0.19 & 0.33 & 0.19 & 0.21 & -0.32 & -0.05 & -0.01 & -0.52 & -0.02 \\
\hline
\end{tabular}

affect the abundances within our sample or that fortuitously, hyperfine structure equally affects the abundances in our sample.

\section{DISCUSSION}

\subsection{Background}

In terms of chemical composition, globular clusters continue to present a series of intriguing questions concerning stellar nucleosynthesis and chemical evolution. This paper examines the pair of clusters M4 and M5 and has confirmed and extended the observations of differences in composition for these clusters of very similar iron abundances. Of the elements we examined up through the Fe-peak, three are more abundant in M4 than in M5: $\mathrm{Si}, \mathrm{Cu}$, and $\mathrm{Zn}$. Differences in $[\mathrm{X} / \mathrm{Fe}]$ are 0.2 to $0.3 \mathrm{dex}$ and might be the same for all three elements. There are abundance differences for the heavy elements $\mathrm{Rb}$ to Th that range from $+0.6(\mathrm{~Pb})$ to $-0.2(\mathrm{Gd})$. It is from these abundance differences that we develop our speculations about stellar nucleosynthesis and chemical evolution.

Determinations of the compositions of globular cluster stars suggest the following chemical history for a cluster. The present population of stars formed from a gas cloud of homogeneous composition; this postulate is necessary to account for the fact that within a given cluster all stars have the same iron abundance (and many other elemental abundances). Within a cluster, there are starto-star differences in the abundances of light elements from $\mathrm{C}$ to $\mathrm{Al}$. Discovery of $\mathrm{O}, \mathrm{Na}$, and $\mathrm{Al}$ abundance differences among turnoff and subgiant stars (e.g., Gratton et al. 2001) proves that a cause is to be found in the cluster's local environment. A possibility, often cited and explored, involves the selective accretion or pollution of material from the winds of AGB stars, particularly highly luminous intermediate-mass AGB stars with hot-bottom convective envelopes capable of nucleosynthesis of $\mathrm{C}$ to $\mathrm{Al}$ (e.g., Cottrell \& Da Costa 1981; Denissenkov et al. 1998; Ventura et al. 2001), although there are quantitative problems with this scenario (Denissenkov \& Herwig 2003; Fenner et al. 2004; Karakas et al. 2006a). Undoubtedly, an additional contribution to the abundance variations involving $\mathrm{C}, \mathrm{N}, \mathrm{Li}$, and possibly $\mathrm{O}$ in red giants is the presence of mixing into the atmosphere of nuclear-processed material from the interior. If the convective mixing, expected to a certain degree of every red giant, is additionally dependent on a stellar property such as rotation, star-to-star differences in composition will result for the red giants.

Given this background, we offer the following idea for the primary origin of the composition differences between M4 and M5. Since the abundances of $\mathrm{Si}, \mathrm{Cu}, \mathrm{Zn}$, and the heavier elements do not show a star-to-star spread across the M4 sample, we suppose that there is no detectable (positive or negative) contribution to their abundances from the polluting stars responsible for the starto-star abundance variations, and, therefore, the abundance differences were present between the clouds forming these two clusters.

In the case of M5 and many other globular clusters, the relative abundances (i.e., the $[\mathrm{X} / \mathrm{Fe}]$ ratios) of the elements unaffected by

TABLE 5

Abundance Ratios [ $\mathrm{X} / \mathrm{Fe}$ ] for $\mathrm{Sr}$ to $\mathrm{Th}$

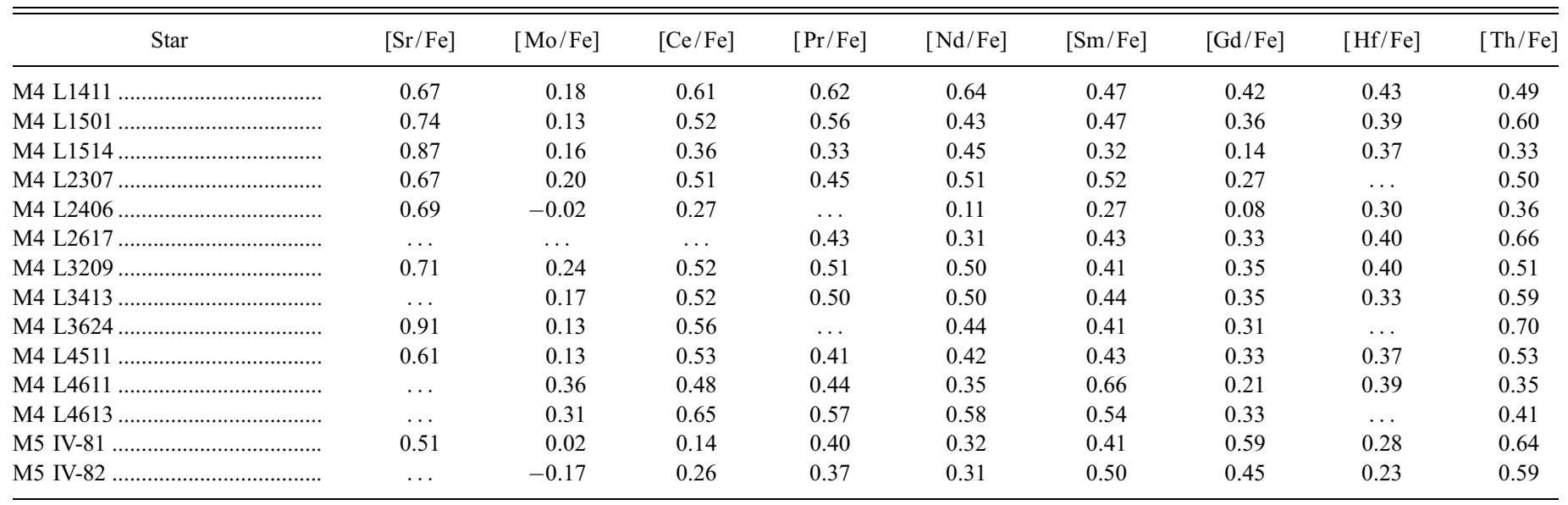


TABLE 6

Abundance Dependences on Model Parameters for M4 L2307

\begin{tabular}{|c|c|c|c|c|}
\hline Species & $T_{\text {eff }}+50$ & $\log g+0.2$ & $\xi_{t}+0.2$ & Total $^{\mathrm{a}}$ \\
\hline$[\mathrm{Si} / \mathrm{Fe}]$ & 0.01 & -0.03 & 0.02 & 0.04 \\
\hline$[\mathrm{Ca} / \mathrm{Fe}]$ & 0.10 & -0.09 & -0.04 & 0.14 \\
\hline$[\mathrm{Sc} / \mathrm{Fe}] \ldots \ldots \ldots$ & 0.02 & 0.01 & -0.08 & 0.09 \\
\hline$[\mathrm{Ti} / \mathrm{Fe}] \ldots \ldots \ldots \ldots \ldots \ldots \ldots$ & 0.12 & -0.08 & 0.01 & 0.14 \\
\hline$[\mathrm{V} / \mathrm{Fe}]$ & 0.13 & -0.07 & -0.01 & 0.15 \\
\hline$[\mathrm{Cr} / \mathrm{Fe}] \ldots \ldots \ldots$ & 0.09 & -0.09 & 0.01 & 0.13 \\
\hline$[\mathrm{Mn} / \mathrm{Fe}] \ldots \ldots \ldots \ldots \ldots \ldots$ & 0.08 & -0.08 & -0.08 & 0.13 \\
\hline$[\mathrm{Fe} / \mathrm{H}] \ldots \ldots \ldots \ldots \ldots$ & -0.01 & 0.04 & -0.03 & 0.05 \\
\hline$[\mathrm{Co} / \mathrm{Fe}]$ & 0.05 & -0.04 & 0.01 & 0.06 \\
\hline$[\mathrm{Ni} / \mathrm{Fe}]$ & 0.03 & -0.04 & 0.01 & 0.05 \\
\hline$[\mathrm{Cu} / \mathrm{Fe}]^{\mathrm{b}}$ & 0.06 & -0.04 & -0.05 & 0.09 \\
\hline$[\mathrm{Zn} / \mathrm{Fe}]^{\mathrm{b}} \ldots \ldots \ldots \ldots \ldots \ldots \ldots \ldots$ & -0.01 & -0.02 & -0.04 & 0.05 \\
\hline$[\mathrm{Sr} / \mathrm{Fe}]$ & 0.10 & -0.09 & 0.05 & 0.15 \\
\hline$[\mathrm{Mo} / \mathrm{Fe}] \ldots \ldots \ldots \ldots \ldots$ & 0.12 & -0.08 & 0.01 & 0.14 \\
\hline$[\mathrm{Ce} / \mathrm{Fe}]$ & 0.04 & 0.01 & 0.03 & 0.05 \\
\hline$[\mathrm{Pr} / \mathrm{Fe}] \ldots \ldots \ldots$ & 0.04 & -0.01 & 0.03 & 0.04 \\
\hline$[\mathrm{Nd} / \mathrm{Fe}]$ & 0.05 & -0.01 & -0.02 & 0.05 \\
\hline$[\mathrm{Sm} / \mathrm{Fe}]^{\mathrm{b}}$ & 0.04 & -0.02 & -0.02 & 0.04 \\
\hline$[\mathrm{Gd} / \mathrm{Fe}]^{\mathrm{b}}$ & 0.03 & -0.01 & 0.03 & 0.05 \\
\hline$[\mathrm{Hf} / \mathrm{Fe}]^{\mathrm{c}}$ & 0.03 & -0.01 & 0.03 & 0.04 \\
\hline$[\mathrm{Th} / \mathrm{Fe}]^{\mathrm{b}}$ & 0.05 & 0.01 & 0.04 & 0.06 \\
\hline
\end{tabular}

a The total value is the quadrature sum of the three individual abundance dependences.

${ }^{\mathrm{b}}$ For elements whose abundances were derived via synthetic spectra, we assumed an equivalent width that produced the final abundance.

${ }^{c}$ Since no abundance was derived for Hf, we assumed an equivalent width that produced the mean cluster abundance.
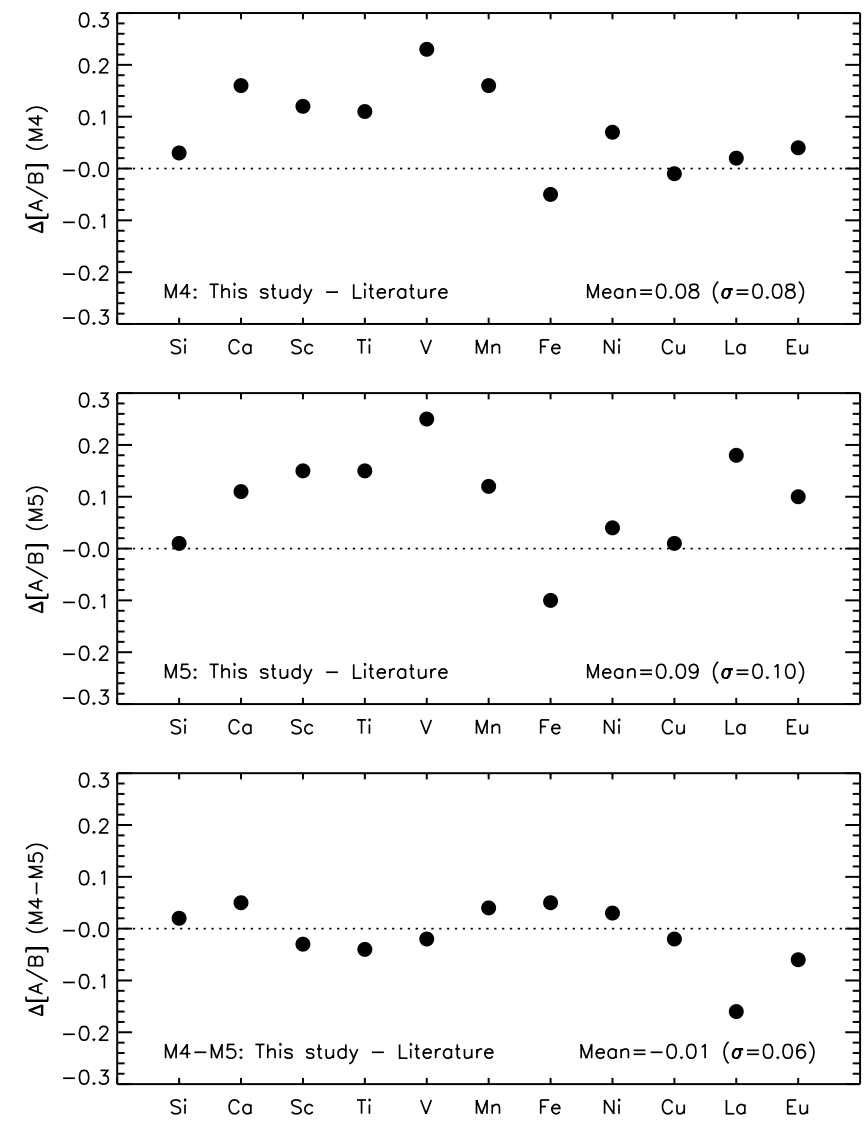

FIG. 5.-Comparison of the mean cluster values $[\mathrm{X} / \mathrm{Fe}]$ and $[\mathrm{Fe} / \mathrm{H}]$ between this study and the literature for M4 (top), M5 (middle), and M4-M5 (bottom). Literature values are from Ivans et al. $(1999,2001)$, except $\mathrm{Mn}$ (Sobeck et al. 2006) and $\mathrm{Cu}$ (Simmerer et al. 2003). The mean differences and standard deviations are shown.
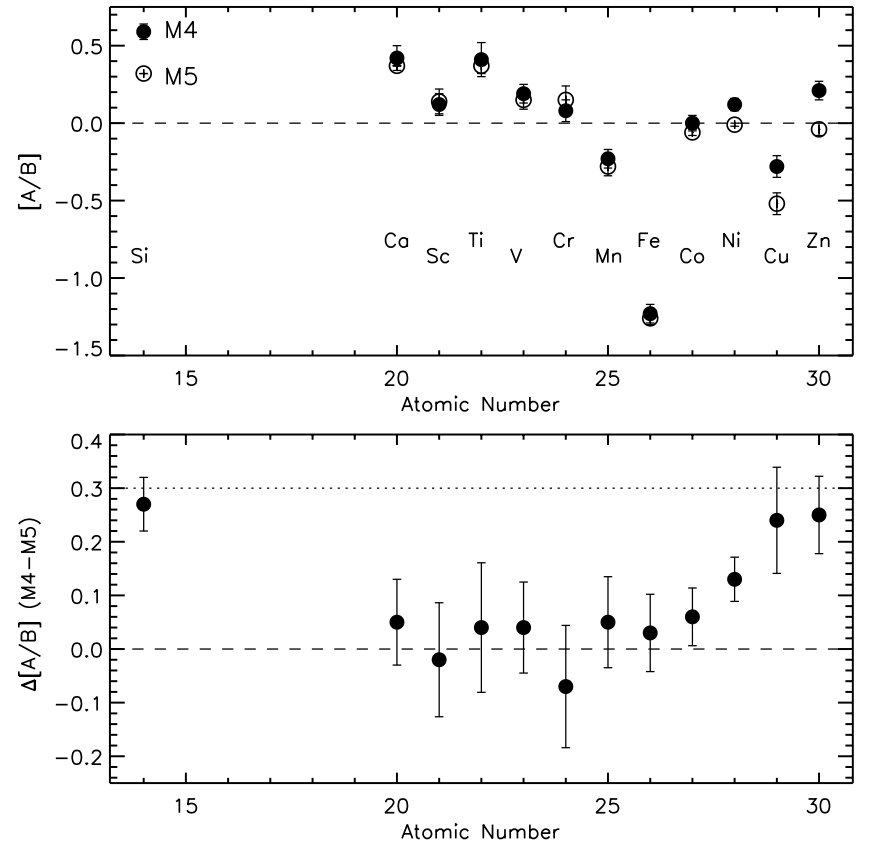

FIG. 6.-Top: $[\mathrm{X} / \mathrm{Fe}]$ for the elements $\mathrm{Si}$ to $\mathrm{Zn}$ as well as $[\mathrm{Fe} / \mathrm{H}]$ for $\mathrm{M} 4$ ( filled circles) and M5 (open blue circles). Bottom: Difference in abundance ratios, $\Delta[\mathrm{X} / \mathrm{Fe}](\mathrm{M} 4-\mathrm{M} 5)$. [See the electronic edition of the Journal for a color version of this figure.]

either mixing in red giants or accretion of pollutants from winds of AGB stars (or other sources) fall within the range exhibited by field stars of the same iron abundance (e.g., Gratton et al. 2004 and Pritzl et al. 2005). This congruence suggests that most globular clusters form from a cloud that underwent the same pattern of chemical evolution that led to the majority of the field halo stars.

The implications of this suggestion are not be thoroughly explored here but two points will be mentioned. One possibility is that field halo stars were constituents of globular clusters but some stars, now field stars, may have been ejected from the present population of clusters. Indeed, the globular clusters Pal 5, NGC 5466, and NGC 6712 exhibit large tidal tails and/or other evidence of severe tidal disruption (de Marchi et al. 1999; Odenkirchen et al. 2001; Belokurov et al. 2006). However, while Pal 5 shows the globular cluster signature of light element abundance variations (Smith et al. 2002), such abundance patterns have yet to be found in field halo stars (Gratton et al. 2000). Alternatively, the process of cluster formation may have resulted in the dispersal of stars rather than a stable cluster. In addition, the suggestion places constraints on the chemical evolution histories of gas clouds from which clusters and field stars formed. In the case of metal-poor clusters including M4 and M5 and field stars of comparable metallicity, chemical evolution was dominated by ejecta from Type II supernovae; Type Ia supernovae have almost certainly not made their distinctive contributions to the composition of the gas cloud. Similarity in $[\mathrm{X} / \mathrm{Fe}]$ ratios implies similarities in the mixing of ejecta with ambient gas over the history of the clouds from which field and cluster stars formed. For elements for which $[\mathrm{X} / \mathrm{Fe}]$ ratios in the ejecta are not very sensitive to the initial mass function or the initial composition (i.e., the $\alpha$-elements), different levels of mixing with pristine gas uncontaminated by $\mathrm{X}$ and $\mathrm{Fe}$, or mixing with gas previously polluted by ejecta from Type II supernovae (the same $[\mathrm{X} / \mathrm{Fe}]$ but different $[\mathrm{Fe} / \mathrm{H}]$ ), will give essentially a single value of $[\mathrm{X} / \mathrm{Fe}]$ at all $[\mathrm{Fe} / \mathrm{H}]$. On the other hand, for elements like $\mathrm{Cu}$ for which the yield is dependent on the initial composition of the massive stars, variations in the mixing processes will lead to a broadening of the $[\mathrm{Cu} / \mathrm{Fe}]$ versus $[\mathrm{Fe} / \mathrm{H}]$ relation. 

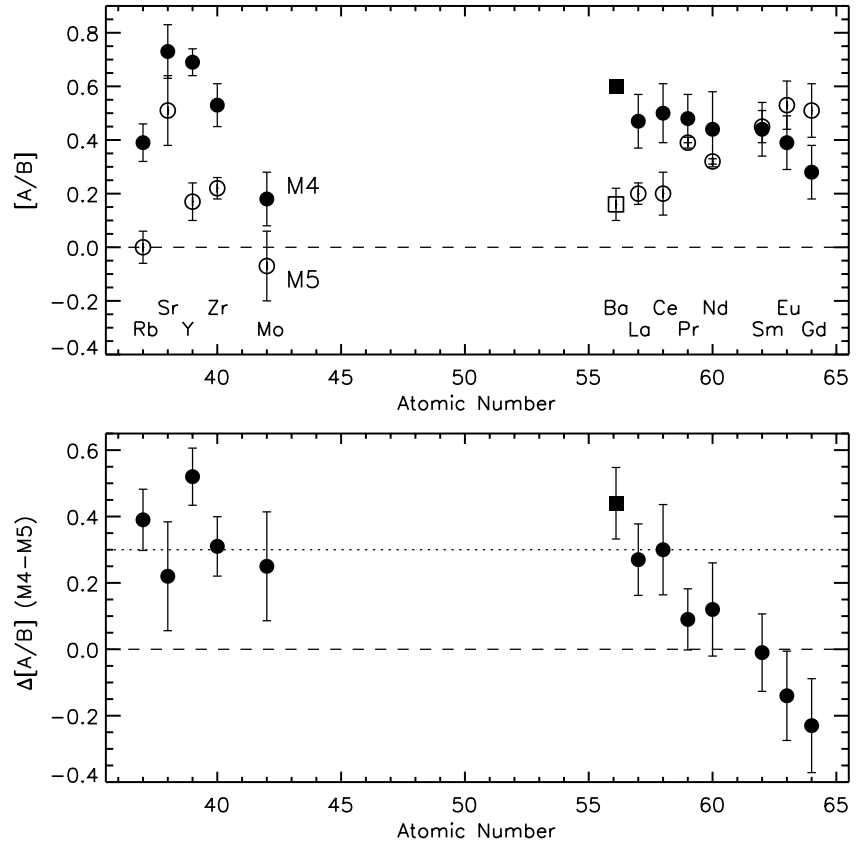

FIG. 7.- Same as Fig. 6, but for the elements Rb to Gd. All data points are from this study and Yong et al. (2008), except Ba which is taken from Ivans et al. (1999, 2001). [See the electronic edition of the Journal for a color version of this figure.]

With this introduction complete, we turn to discussion of the abundance differences between M4 and M5.

\subsection{The s- and r-Process Mix: Ba to Th}

With the usual attribution of $\mathrm{Ba}$ to the $s$-process and $\mathrm{Eu}$ to the $r$-process, the higher $[\mathrm{Ba} / \mathrm{Fe}]$ and the lower $[\mathrm{Eu} / \mathrm{Fe}]$ for $\mathrm{M} 4$ relative to M5 indicates that the M4 stars formed from gas that was enriched in $s$-process products but deficient in $r$-process products relative to the mixtures in the gas that formed the M5 stars. This attribution may be subject to quantitative analysis with two assump-
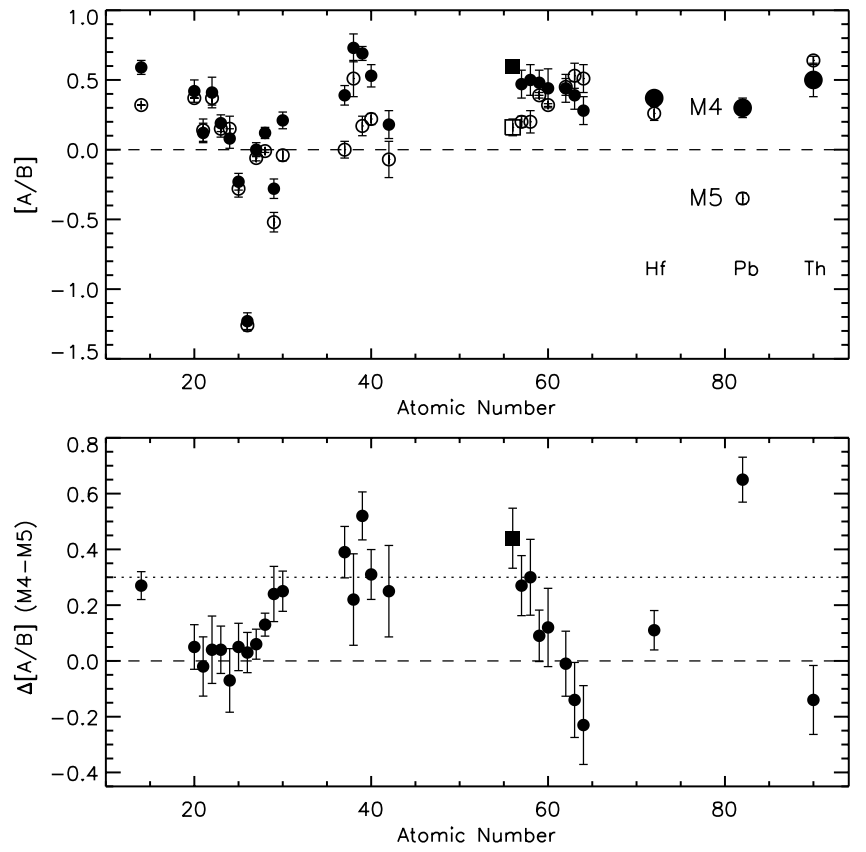

FIG. 8.- Same as Figs. 6 and 7, but for all elements. The heavy neutron-capture elements $\mathrm{Hf}, \mathrm{Pb}$, and $\mathrm{Th}$ are highlighted in red. [See the electronic edition of the Journal for a color version of this figure.]

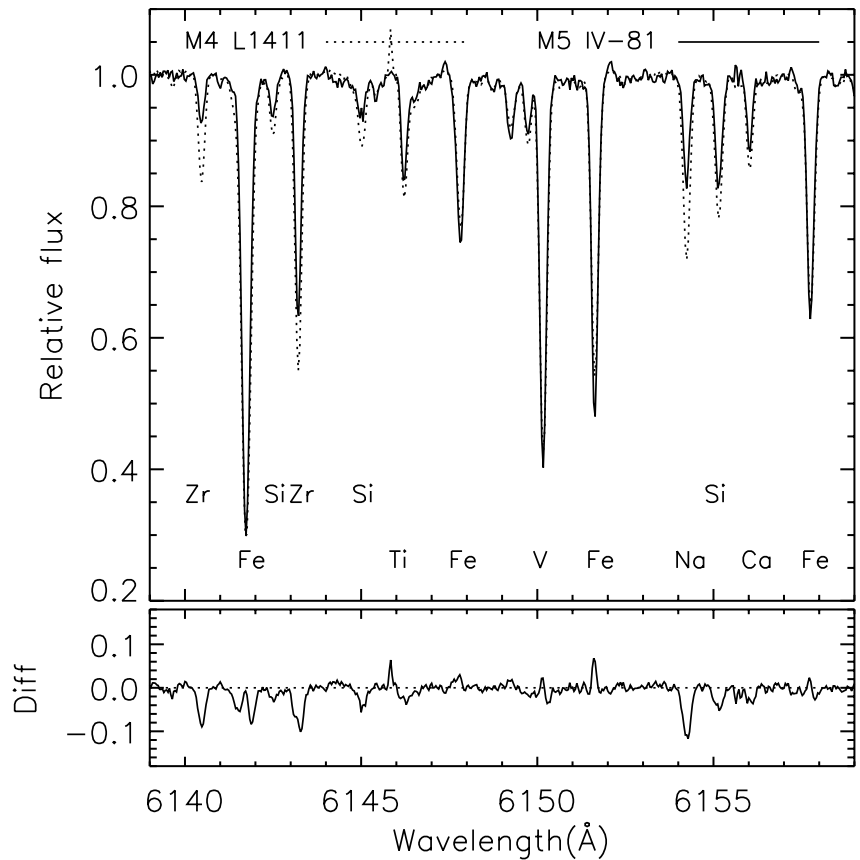

FIG. 9.- Top: Observed spectra for M4 L1411 (dotted blue line) and M5 IV-81 (solid black line). Various lines within the wavelength region are identified. The bottom panel shows the difference M4 L1411 - M5 IV-81 after interpolating and rebinning the spectra. [See the electronic edition of the Journal for a color version of this figure.]

tions. It has been shown that relative abundances of $r$-process products in the interval Ba to Ir in $r$-process-enriched stars (e.g., Sneden et al. 1996; Westin et al. 2000; Christlieb et al. 2004) and in the globular cluster M15 (Sneden et al. 1997, 2000; Otsuki et al. 2006) closely follows the distribution of $r$-process products derived from solar system abundances. This observation that the $r$-process from Ba to Ir is "universal" remains without convincing theoretical explanation. Observations show that universality does not extend to the lighter elements - $\mathrm{Rb}$ to Mo are the

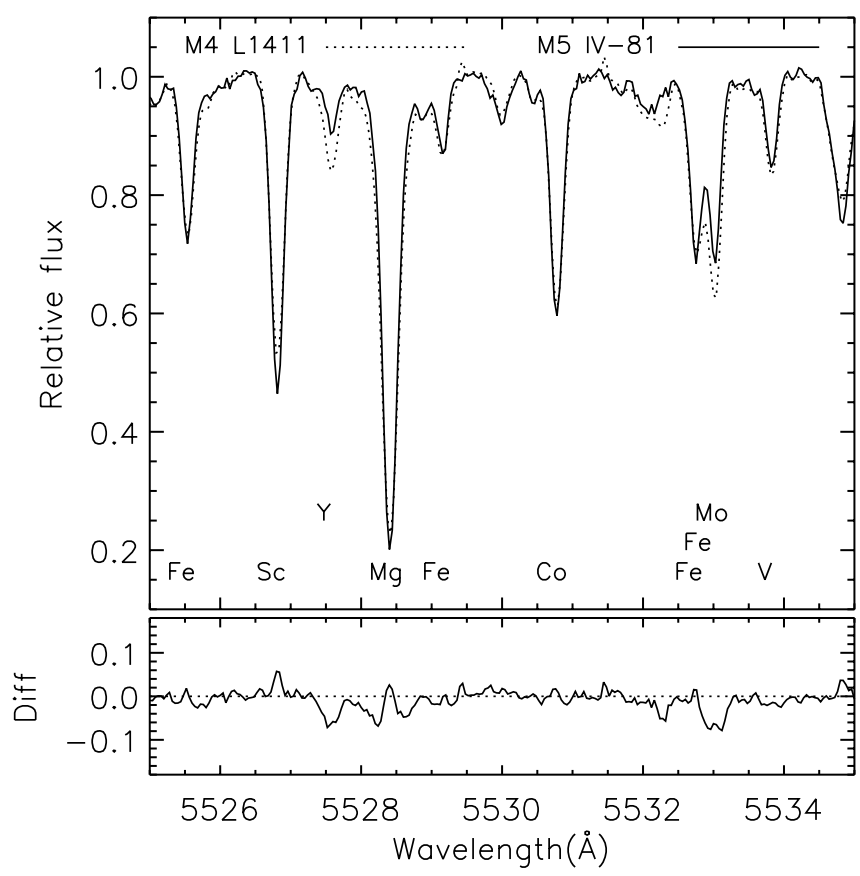

FIG. 10.- Same as Fig. 9, but for a different wavelength region. [See the electronic edition of the Journal for a color version of this figure.] 


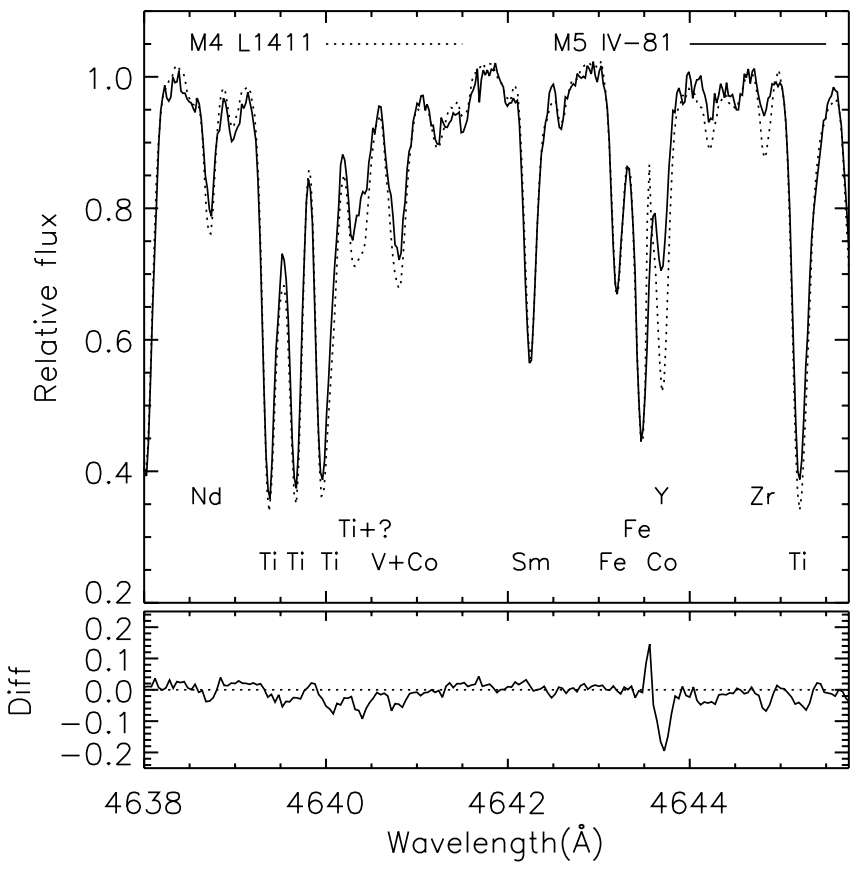

FIG. 11.- Same as Fig. 9, but for a different wavelength region. [See the electronic edition of the Journal for a color version of this figure.]

observable elements. Our first assumption is that the $r$-process contributions to M4 and M5 are scaled versions of the solar $r$-process abundances. The second assumption is that the $s$-process contributions are scaled versions of the solar $s$-process abundances. Resolution of the solar abundances into $r$ - and $s$-process contributions is taken from Simmerer et al. (2004). These assumptions are not validated by observations unlike the assumption about the $r$-process.

For M4 and M5, we adopted their mean abundance ratios $[\mathrm{X} / \mathrm{Fe}]$. We then took linear combinations of the solar $s$ - and $r$-process abundances (adopting $[\mathrm{Fe} / \mathrm{H}]=-1.25$ ) exploring the full range of parameter space (i.e., considering values from 0.01 to 1.00 times the solar $s$ - and $r$-process abundances). For each combination of $s$-and $r$-process abundances, we compared the predicted abundance ratios with the observed abundance ratios. For each cluster, we located the optimal scaling factors for the $s$ - and $r$-process. When considering the elements from $\mathrm{Ba}$ to $\mathrm{Hf}$, we find $s=0.188$ and $r=0.135$ for M4 and $s=0.060$ and $r=0.205$ for M5 where $s$ and $r$ are the scaling factors of the solar $s$ - and $r$ process abundances, respectively. For both clusters, the predicted and measured abundances are in very good agreement (see Fig. 12). For M4 we find $\Delta[\mathrm{X} / \mathrm{Fe}]_{\text {predicted-observed }}=0.04(\sigma=0.05)$ and for M5 we find $\Delta[\mathrm{X} / \mathrm{Fe}]=0.02(\sigma=0.04)$.

For both clusters, the abundances of Th lie above the predictions, albeit by similar amounts. The ratio of $\mathrm{Th}$ to $\mathrm{Eu}$ is identical in both clusters, $[\mathrm{Th} / \mathrm{Eu}]=0.11$. Since $\mathrm{Th}$ and $\mathrm{Eu}$ are produced exclusively by the $r$-process, the identical [ $\mathrm{Th} / \mathrm{Eu}$ ] ratios in M4 and M5 indicate that the $r$-process universality extends to Th in both clusters. Furthermore, the identical [ $\mathrm{Th} / \mathrm{Eu}$ ] ratios suggests that no differential decay of Th has occurred and that these two clusters have essentially identical ages. (An uncertainty of 0.1 dex in the $[\mathrm{Th} / \mathrm{Eu}]$ ratio would result in an age uncertainty of roughly 3 billion yr.)

For both clusters, the observations of $\mathrm{Pb}$ fall below the predictions with M4 lying closer to the prediction than M5 owing to the different abundances, $[\mathrm{Pb} / \mathrm{Fe}]=0.30$ in $\mathrm{M} 4$ and $[\mathrm{Pb} / \mathrm{Fe}]=$ -0.35 in M5. As mentioned, the $\mathrm{Pb}$ abundance in M5 is typical of field halo stars at the clusters' metallicities. The cluster-to-cluster

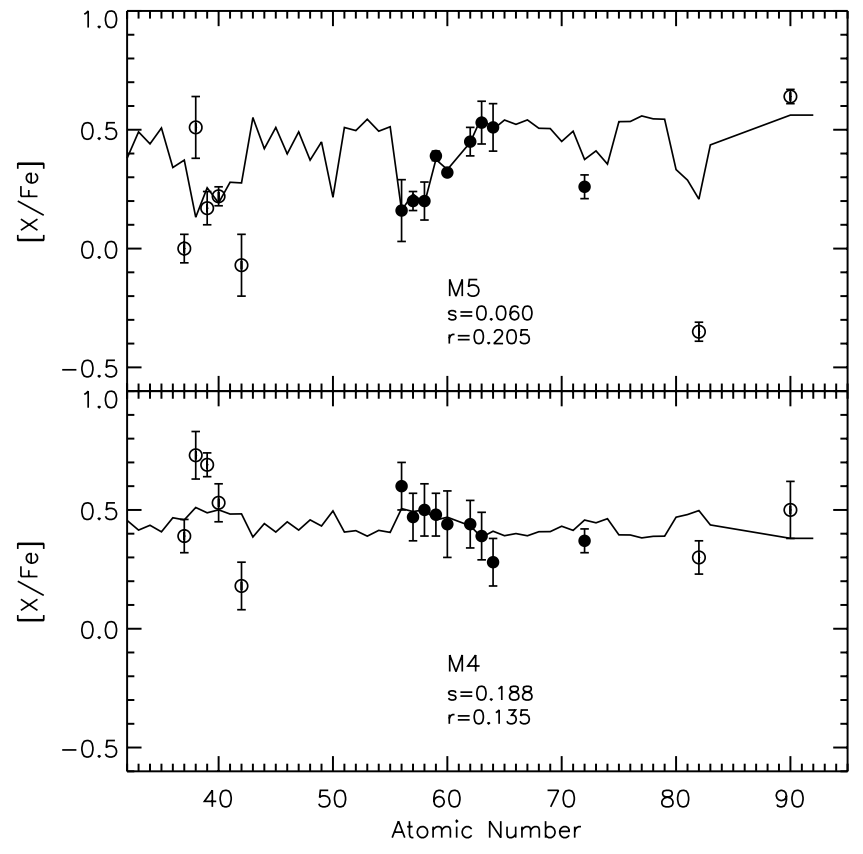

FIG. 12.-Abundance ratios [X/Fe] for $\mathrm{Rb}$ to Th in M5 (top) and M4 (bottom). In both panels we show the best-fit predictions to the elements from Ba to $\mathrm{Hf}$ ( filled circles) using scaling factors $s$ and $r$ which were multiplied by the solar $s$-process and $r$-process abundances, respectively.

differences between the observed and predicted $\mathrm{Pb}$ abundances indicate that the $s$-process similarities break down for $\mathrm{Pb}$. In the Sun, more than $50 \%$ of ${ }^{208} \mathrm{~Pb}$, the most abundant isotope, is due to the so-called strong component (e.g., Clayton \& Rassbach 1967; Käppeler et al. 1989), where low-mass metal-poor AGB stars are the likely site of this component. Therefore, the differences between the observed and predicted $\mathrm{Pb}$ abundances in M4 and M5 provide a hint that the sources of the $s$-process, in particular the strong component, differ between these clusters.

Had we included all elements from $\mathrm{Ba}$ to Th in determining the scaling factors for the solar $s$ - and $r$-process abundances, we would have obtained very similar values (M4: $s=0.175, r=0.138$, and M5: $s=0.053, r=0.210$ ) as those obtained when fitting the $\mathrm{Ba}$ to $\mathrm{Hf}$ abundances. These new predictions again provide a poor fit to $\mathrm{Pb}$ and $\mathrm{Th}$.

The mix of $r$-and $s$-process contributions for M5 is typical of the mix found in halo field stars but the mix for M4 is quite atypical. This is well shown when considering the ratio of the La to Eu abundances. Inspection of Figure 7 in Simmerer et al. (2004) shows that $\mathrm{M} 5, \log \epsilon(\mathrm{La} / \mathrm{Eu})=0.28$, is representative of field stars at the cluster's metallicity. However, M4, $\log \epsilon(\mathrm{La} / \mathrm{Eu})=$ 0.70 , is in the far tail of the distribution of $\mathrm{La} / \mathrm{Eu}$ ratios for field stars and close to the stars labeled as $s$-process enriched. Yet, as our fits to the abundance patterns show, the $s$-process in M4 has the same pattern of abundances as in M5 and the Sun. Thus, no new site for $s$-process operation is needed to account for the M4 - M5 differences, at least for the elements Ba to Hf. We presume that AGB stars are the active site. Yet, the difference in $s$-process pollution between M4 and M5 might suggest that M4 originated in a different part of the Galaxy to M5 and local halo stars.

\section{3. $\mathrm{Cu}$ to $\mathrm{Zr}$}

The successful dissection of the $\mathrm{Ba}-\mathrm{Ir}$ abundances into different mixing fractions of solar-like $r$ - and $s$-process abundance patterns cannot be easily extended to lighter elements in the $\mathrm{Cu}-\mathrm{Zr}$ interval. This is likely attributable to the appearance of at least one additional process of stellar nucleosynthesis. 
One expects the weak $s$-process to contribute to the elements $\mathrm{Cu}$ to $\mathrm{Zr}$ as revealed from the fit to the solar abundances (e.g., Käppeler et al. 1989). This neutron capture process occurs in massive stars in the He-burning and the C-burning shells with products ejected without substantial modification in the supernova explosion. At initial metallicities $[\mathrm{Fe} / \mathrm{H}] \sim-1$, the contributions from the He-burning shell appear to be more important than from the C-burning shell. This $s$-process does not provide significant yields beyond the cross-section bottleneck at the neutron magic number $N=50$ and, thus, the Ba-Ir interval is unaffected by this contribution from massive stars. Predicted yields for this weak $s$-process are sketched by Pignatari \& Gallino (2007). It is also below $[\mathrm{Fe} / \mathrm{H}] \sim-1$ that the weak $s$-process may become so ineffective that explosive Si-burning may replace it as the source of $\mathrm{Cu}, \mathrm{Zn}$, and other weak $s$-process products.

The $r$-process, whose site is probably the deep interior of Type II supernovae from massive stars, is likely to contribute also to the $\mathrm{Cu}-\mathrm{Zr}$ interval, in part or in whole. Unfortunately, there are not even qualitative predictions about these $r$-process yields. Abundance analyses of very $r$-process-enriched stars by Sneden and colleagues have shown a star-to-star variation in these lighter elements- $\mathrm{Ga}$ to $\mathrm{Ag}-$ in their abundance ratios with respect to the abundances of the heavier elements from $\mathrm{Ba}$ and up. These variations have been ascribed to "a second $r$-process," but may at least in part reflect a weak $s$-process contribution from massive stars, also the likely site of the $r$-process(es). (Recall that we have discounted the possibility that Type Ia supernovae are contributing to the chemical evolution of M4 and M5 and field stars of comparable or lower metallicity.)

As expected, Figure 12 shows that predictions based on the scaling factors for the solar $s$ - and $r$-process abundances that match the elements from $\mathrm{Ba}$ to $\mathrm{Hf}$ provide a poor fit to the elements from $\mathrm{Rb}$ to Mo. Had we included all elements from $\mathrm{Rb}$ to $\mathrm{Th}$ in the fit, the scaling factors would be essentially unchanged (M4: $s=$ $0.060, r=0.198$, and M5: $s=0.188, r=0.138$ ), and the fit to the elements $\mathrm{Rb}$ to Mo would remain poor.

\subsection{The Case of $\mathrm{Si}$}

An overabundance of $\alpha$-elements is commonly taken as the signature of gas contaminated to a major degree by ejecta of Type II supernovae. Here, the $\alpha$-element directory includes $\mathrm{O}, \mathrm{Mg}, \mathrm{Si}, \mathrm{S}$, and $\mathrm{Ca}$, with $\mathrm{Ti}$ as an honorary member according to observers. $\mathrm{Ca}$ and Ti have very similar abundances in M4 and M5, but Si is definitely overabundant in M4 with respect to M5 (Fig. 6 and Table 4). In M4, the overabundance of $\mathrm{Mg},[\mathrm{Mg} / \mathrm{Fe}]=0.56(\sigma=$ 0.05 ) from 12 stars (Yong et al. 2008), is similar to that of $\mathrm{Si}$, $[\mathrm{Si} / \mathrm{Fe}]=0.58(\sigma=0.08)$. This contradicts findings by Ivans et al. (2001), who found that stars in M4 have Si abundances about 0.2 dex higher than $\mathrm{Mg}$. A more careful investigation of the difference in $[\mathrm{Mg} / \mathrm{Si}]$ ratios in $\mathrm{M} 4$ by different authors would need to account for systematic differences.

Both clusters exhibit the classic signature of $\alpha$-elements in metal-poor stars- $[\alpha / \mathrm{Fe}] \simeq 0.3 \mathrm{dex}$ - but this signature is different in its details, as just noted. There is no obvious explanation for the enhanced $\mathrm{Si}$ abundances in M4. Si and $\mathrm{Ca}$ (as well as $\mathrm{S}$ and $\mathrm{Ar}$ ) are all produced by incomplete explosive Si burning and explosive $\mathrm{O}$ burning in massive stars. Therefore, any Si excess in M4 relative to M5 should be accompanied by a similar Ca excess.

\subsection{Comments on $\mathrm{Cu}$ and $\mathrm{Zn}$ Nucleosynthesis}

Pioneering measurements of $\mathrm{Cu}$ and $\mathrm{Zn}$ in field stars showed that between $-2.5 \leq[\mathrm{Fe} / \mathrm{H}] \leq 0.0,[\mathrm{Cu} / \mathrm{Fe}]$ declined with decreasing metallicity, whereas [Zn/Fe] was constant and solar (Peterson 1981; Gratton \& Ortolani 1988; Sneden \& Crocker 1988; Sneden et al.
1991). Although a large amount of data is now available for these elements, the nucleosynthetic origins of $\mathrm{Cu}$ and $\mathrm{Zn}$ remain unclear with both elements likely requiring multiple production sites and metallicity dependent yields. For Cu, Type Ia supernovae (Matteucci et al. 1993; Mishenina et al. 2002) and massive stars (Timmes et al. 1995; Romano \& Matteucci 2007) are proposed to be the dominant producers with low-mass AGB stars believed to provide only a minor contribution (Matteucci et al. 1993). For Zn, massive stars (Timmes et al. 1995) and Type Ia supernovae (Matteucci et al. 1993) have been suggested as being the major producers. Other potential sources of $\mathrm{Zn}$ include the weak $s$-process in massive stars and the main $s$-process in low-mass AGB stars (Matteucci et al. 1993), and at very low metallicity, the $s$-process or $r$-process in massive stars (Umeda \& Nomoto 2002; Heger \& Woosley 2002).

The abundances measured in M4 and M5 offer additional insight into the production sites of $\mathrm{Cu}$ and $\mathrm{Zn}$. These metal-poor globular clusters were formed early in the history of our Galaxy, before Type Ia supernovae contributed to their chemical evolution. Therefore, our measurements confirm that stars other than Type Ia supernovae must produce significant amounts of $\mathrm{Cu}$ and $\mathrm{Zn}$ at low metallicities. Abundance differences of roughly 0.3 dex are found for the $\alpha$-element $\mathrm{Si}$ (produced primarily in massive stars) as well as the $s$-process elements (produced in AGB stars or massive stars). In light of the abundance differences for $\mathrm{Cu}$ and $\mathrm{Zn}$ in these two clusters, we now examine the synthesis of $\mathrm{Cu}$ and $\mathrm{Zn}$ in massive stars and AGB stars based on recent theoretical models.

\subsubsection{Massive Star Production}

Both the stable isotopic nuclei of $\mathrm{Cu}$ and the five of $\mathrm{Zn}$ are produced, at low metallicities $([\mathrm{Fe} / \mathrm{H}]<-1)$, only by the complete explosive Si burning, i.e., in the deepest regions of the exploding star where the peak temperature exceeds $5 \times 10^{9} \mathrm{~K}$ and therefore matter can reach the nuclear statistical equilibrium (Chieffi \& Limongi 2004). The striking similarities of the relative abundances of the elements between $\mathrm{Ca}$ and $\mathrm{Ni}$ seem to clearly indicate that the core collapse supernovae responsible for the bulk production of the nuclei in this range are very similar in M4 and M5. In this respect $\mathrm{Cu}$ and $\mathrm{Zn}$ should also behave similarly (because of their production site) but there is a "caveat": they are less abundant (relative to hydrogen) than $\mathrm{Co}$ and $\mathrm{Ni}$, and hence they would be the first elements within the Fe peak nuclei to be modified by the passage of a neutron flux.

In massive stars, He and $\mathrm{C}$ burning can lead to activation of the ${ }^{22} \mathrm{Ne}(\alpha, n){ }^{25} \mathrm{Mg}$ neutron source whose high neutron densities are responsible for synthesizing $s$-process elements up to $\mathrm{Sr}$ (Busso et al. 1999). If the weak $s$-process is solely responsible for the enhanced $\mathrm{Cu}$ and $\mathrm{Zn}$ in M4, we would expect the abundances of $\mathrm{Rb}$ relative to nearby elements such as $\mathrm{Sr}, \mathrm{Y}$, and $\mathrm{Zr}$ to also differ between these clusters (Tomkin \& Lambert 1983; Lambert et al. 1995; Abia et al. 2001). Observation support for this scenario comes from enhanced $\mathrm{Rb}$ abundances in luminous Galactic $\mathrm{OH} / \mathrm{IR}$ stars of solar metallicity (García-Hernández et al. 2006) that are matched by theoretical models (van Raai et al. 2008). However, Yong et al. (2008) showed that in M4 and M5 the ratio [Rb/Zr] was identical within the measurement uncertainties, and therefore it may be difficult to ascribe any difference in $\mathrm{Cu}$ and $\mathrm{Zn}$ solely to the weak $s$-process, unless the production of $[\mathrm{Rb} / \mathrm{Zr}]$ has a metallicity dependence and is not significant at low metallicity. As discussed above, no comprehensive predictions are presently available.

\subsubsection{AGB Production}

Models presented in Karakas et al. (2008) indicate that $\mathrm{Zn}$, in general, is not altered by AGB nucleosynthesis. $\mathrm{Zn}$ can be produced 
TABLE 7

Mg Isotope Ratios

\begin{tabular}{|c|c|}
\hline Star & ${ }^{24} \mathrm{Mg}:{ }^{25} \mathrm{Mg}:{ }^{26} \mathrm{Mg}$ \\
\hline 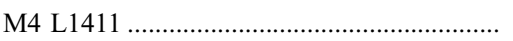 & $80: 10: 10$ \\
\hline M4 L1501 …… & \\
\hline M4 L1514 … & $80: 10: 10$ \\
\hline 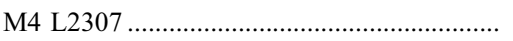 & $72: 14: 14$ \\
\hline 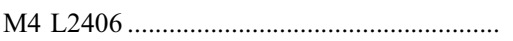 & $\ldots$ \\
\hline 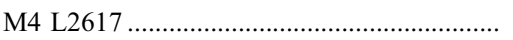 & $74: 13: 13$ \\
\hline 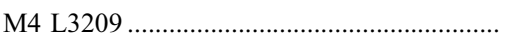 & $74: 13: 13$ \\
\hline M4 L3413 ................. & $80: 10: 10$ \\
\hline M4 L3624 ............. & $78: 11: 11$ \\
\hline 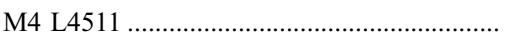 & $80: 10: 10$ \\
\hline 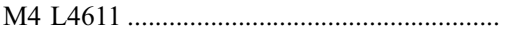 & $80: 10: 10$ \\
\hline 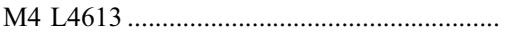 & $78: 11: 11$ \\
\hline M5 IV-81 „.. & $80: 10: 10$ \\
\hline 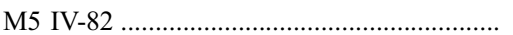 & $78: 11: 11$ \\
\hline
\end{tabular}

in the most massive, lowest metallicity AGB stars, with the largest abundance predicted to be $[\mathrm{Zn} / \mathrm{Fe}] \sim 0.4$ dex in a $5 M_{\odot}$, $Z=0.004$ model. The total expelled mass of $\mathrm{Zn}$ in this case is small $\left(\sim 10^{-6} M_{\odot}\right)$. Preliminary results for $\mathrm{Cu}$ suggest that neutroncapture nucleosynthesis in intermediate-mass low-metallicity AGB stars could be an extra production site for this element. Both stable isotopes of $\mathrm{Cu}$ are predicted to be enhanced in the He-shell of 4 to $8 M_{\odot}$ AGB stars, with the final elemental $\mathrm{Cu}$ surface abundance estimated to be $[\mathrm{Cu} / \mathrm{Fe}] \sim 0.8 \mathrm{dex}$ in a $5 M_{\odot}, Z=0.004$ model.

The results for $\mathrm{Zn}$ agree with the Travaglio et al. (2004) predictions that only $2.4 \%$ of elemental $\mathrm{Zn}$ comes from the $s$-process; however, the results for $\mathrm{Cu}$ are less clear, for which Travaglio et al. (2004) estimate that $5.2 \%$ of $\mathrm{Cu}$ is produced in AGB stars. We would need to include updated AGB yields of $\mathrm{Cu}$ and $\mathrm{Zn}$ in a chemical evolution model to study the consistency with the Travaglio et al. (2004) results, as well as to determine just how efficient intermediate-mass AGB production really is, along with an exhaustive study of the many uncertainties affecting the AGB nucleosynthesis models. For example, one of the largest uncertainties is the mass-loss law used in the calculations, as this determines the number of third dredge-up mixing episodes. If the number of mixing episodes were to be halved, then about 4 times less $\mathrm{Cu}$ would be produced in the $5 M_{\odot}$ example given above.

If $5 M_{\odot}$ metal-poor AGB stars are solely responsible for the enhanced $\mathrm{Cu}$ and $\mathrm{Zn}$ in $\mathrm{M} 4$, we would expect differences in the abundances of the heavy Mg isotopes between M4 and M5 (Karakas \& Lattanzio 2003; Karakas et al. 2006b). Observations of high ${ }^{26} \mathrm{Mg} /{ }^{24} \mathrm{Mg}$ ratios in field and cluster stars confirm the AGB yields (Gay \& Lambert 2000; Yong et al. 2003a, 2003b). Although the resolution is less than ideal $(R=55,000$ in this study compared with $R \geq 90,000$ in our previous studies of $\mathrm{Mg}$ isotope ratios in giant stars in globular clusters), we have measured the $\mathrm{Mg}$ isotope ratios using the same methods as Yong et al. (2003a, 2006a). The ratios are given in Table 7 and examples of synthetic spectra fits are shown in Figure 13. Given the resolution, we adopt ${ }^{25} \mathrm{Mg}={ }^{26} \mathrm{Mg}$ in the reported ratios, and therefore we regard these results as preliminary because our analyses of stars in other globular clusters have showed that ${ }^{25} \mathrm{Mg}$ and ${ }^{26} \mathrm{Mg}$ can behave independently. The uncertainties in the ratios are $b \pm 5$ and $c \pm 5$ when expressing the ratio as ${ }^{24} \mathrm{Mg}:{ }^{25} \mathrm{Mg}:{ }^{26} \mathrm{Mg}=$ $(100-b-c): b: c$. Nevertheless, these preliminary results provide important additional information into the chemical evolution of these clusters. Within M4 and M5, the Mg isotope ratios are constant, in contrast to what is seen in the more metal-poor globular clusters NGC 6752 and M13 (Shetrone 1996; Yong et al.

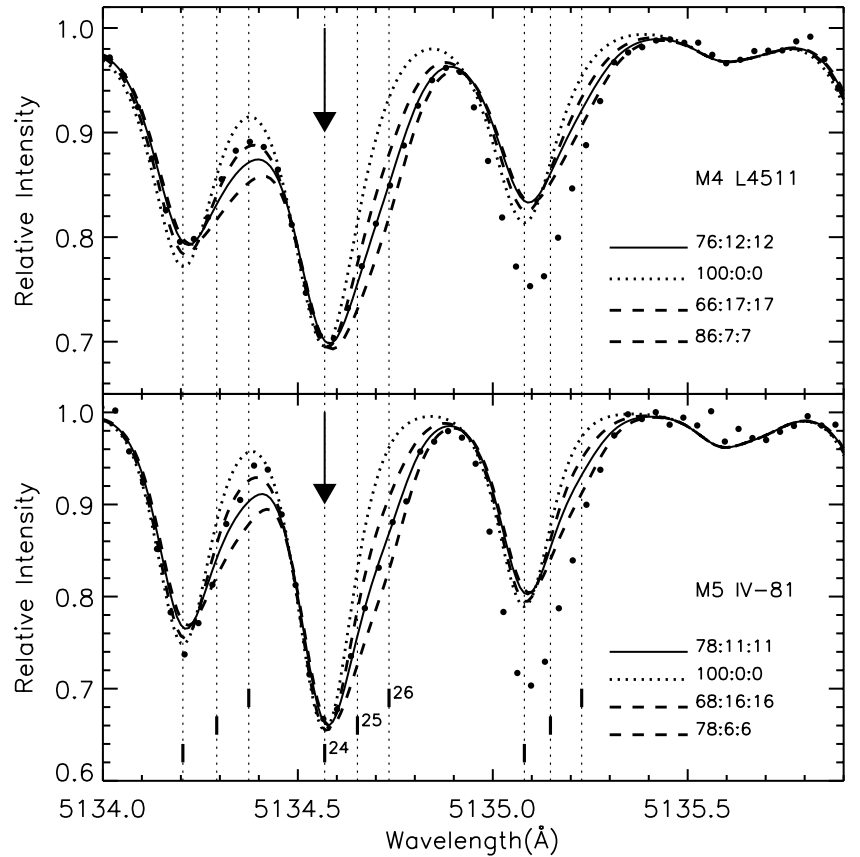

FIG. 13. - Spectra of M4 L4511 (top) and M5 IV-81 near the 5134.6 ̊ MgH line. The positions of ${ }^{24} \mathrm{MgH},{ }^{25} \mathrm{MgH}$, and ${ }^{26} \mathrm{MgH}$ are marked by dashed lines. The filled circles represent the observed spectra, the best fit is shown by the solid line, and unsatisfactory ratios are also shown. [See the electronic edition of the Journal for a color version of this figure.]

2003a, 2006a). Given the small amplitude $[\mathrm{Al} / \mathrm{Fe}]$ variation in our sample $\left(\Delta[\mathrm{Al} / \mathrm{Fe}]_{\mathrm{M} 4}=0.23\right.$ compared with $\Delta[\mathrm{Al} / \mathrm{Fe}]_{\mathrm{NGC6752}}=$ 1.06), it is not surprising that the $\mathrm{Mg}$ isotope ratios show a smaller variation in M4 than in NGC 6752 (and M13). In both M4 and M5, the ratios are very similar with a typical cluster ratio comparable to the solar value, ${ }^{24} \mathrm{Mg}:{ }^{25} \mathrm{Mg}:{ }^{26} \mathrm{Mg}=80: 10: 10$. Even at our resolution, the isotope ratios ${ }^{25} \mathrm{Mg} /{ }^{24} \mathrm{Mg}$ and ${ }^{26} \mathrm{Mg} /{ }^{24} \mathrm{Mg}$ in M4 and M5 exceed values measured in field halo stars at the clusters' metallicities (Gay \& Lambert 2000; Yong et al. 2003b; Meléndez \& Cohen 2007) and predictions from chemical evolution models (Alibés et al. 2001; Fenner et al. 2003). For example, the halo dwarf $\mathrm{Gmb} 1830$ has $[\mathrm{Fe} / \mathrm{H}]=-1.30$ and ${ }^{24} \mathrm{Mg}:{ }^{25} \mathrm{Mg}:{ }^{26} \mathrm{Mg}=94: 3: 3$ (Tomkin \& Lambert 1980). Therefore, the clouds from which M4 and M5 formed were not like the clouds from which halo stars like Gmb 1830 formed. In summary, the Mg isotope ratios in M4 and M5 do not support the scenario in which intermediate AGB stars are responsible for the $\mathrm{Cu}$ and $\mathrm{Zn}$ abundance differences between these clusters. For both clusters the isotope ratios exceed the values found in field stars at the same metallicity.

Further constraints on the AGB contribution to the $\mathrm{Cu}$ abundance differences in M4 and M5 come from the most massive globular cluster, $\omega$ Centauri. In $\omega$ Cen, Cunha et al. (2002) found a roughly constant ratio $[\mathrm{Cu} / \mathrm{Fe}] \simeq-0.5$ as $[\mathrm{Fe} / \mathrm{H}]$ ranged from -2.0 to -0.8 in their sample of 40 stars. The same sample was previously analyzed by Norris \& Da Costa (1995), who found that the abundance ratios $[\mathrm{X} / \mathrm{Fe}]$ for $s$-process elements increased as $[\mathrm{Fe} / \mathrm{H}]$ increases. Therefore, the low-mass AGB stars presumably responsible for the increasing $[s$-element $/ \mathrm{Fe}]$ as $[\mathrm{Fe} / \mathrm{H}]$ increases do not synthesize $\mathrm{Cu}$. However, $\omega \mathrm{Cen}$ exhibits a number of traits not found in other globular clusters. Detailed chemical evolution models such as those performed by Fenner et al. (2004) and Renda et al. (2004) will help unravel the origin of the $\mathrm{Cu}$ and $\mathrm{Zn}$ abundance differences in M4 and M5 as well as the synthesis of $\mathrm{Cu}$ and $\mathrm{Zn}$ in metal-poor stars. 


\subsection{Physical and Kinematic Differences between M4 and M5}

Finally, we briefly consider the physical and kinematic parameters for M4 and M5. Within the Galactic globular clusters, M4 has a typical luminosity (i.e., mass), $M_{V}=-7.20$, while M5 is one of the more luminous (i.e., massive) clusters, $M_{V}=-8.81$ (Harris 1996). ${ }^{3}$ The space velocities for these two clusters are considerably different. Dinescu et al. (1999) have shown that M5 has a very large apocentric radius $\left(R_{a}=35.4 \mathrm{kpc}\right)$, while M4 has a very small apocentric radius $\left(R_{a}=5.9 \mathrm{kpc}\right)$. Given that M4's orbit is confined to the inner disk and bulge, it would be of great interest to measure the abundances of $\mathrm{Si}, \mathrm{Cu}, \mathrm{Zn}$, and $s$-process elements in a sample of inner disk and bulge stars at the metallicity of M4. Perhaps the elevated abundances of $\mathrm{Si}, \mathrm{Cu}, \mathrm{Zn}$, and $s$-elements in M4 are representative of stars born at small Galactocentric radii. However, we note that Fulbright et al. (2007) measured the abundances of $\mathrm{Si}, \mathrm{Ca}$, and $\mathrm{Ti}$ in bulge giants including four with $-1.5 \leq[\mathrm{Fe} / \mathrm{H}] \leq-1.0$. All have $[\mathrm{Si} / \mathrm{Fe}]$ ratios comparable to $[\mathrm{Ca} / \mathrm{Fe}]$ and $[\mathrm{Ti} / \mathrm{Fe}]$. Two stars below $[\mathrm{Fe} / \mathrm{H}]=-1$ in the Fulbright et al. (2007) sample have high $[\mathrm{Al} / \mathrm{Fe}]$ and $[\mathrm{Na} / \mathrm{Fe}]$ along with low $[\mathrm{O} / \mathrm{Fe}]$, the abundance signature of hydrogen burning at high temperatures seen in globular clusters. Indeed, they speculate that these two stars may be members of the bulge globular cluster NGC 6522. Abundances of $s$-process elements were measured by McWilliam \& Rich (1994) in three bulge giants with $[\mathrm{Fe} / \mathrm{H}] \leq-0.8$, none of which showed $s$-process enrichments. Therefore, the enhanced abundances of Si and $s$-elements in M4 may not be representative of stars born at small Galactocentric radii.

\section{CONCLUDING REMARKS}

In this paper we present abundance ratios $[\mathrm{X} / \mathrm{Fe}]$ for a large number of $\alpha$-, Fe-peak, $s$-process, and $r$-process elements for 12 bright giants in the globular cluster M4 and 2 bright giants of the globular cluster M5. This comprehensive abundance analysis is only possible due to the large wavelength coverage, high resolution, and very high $\mathrm{S} / \mathrm{N}$ spectra. For all elements in this study, we find no evidence for star-to-star abundance variations in either cluster. We confirm and extend on previous results for these clusters by showing that (1) for the elements from $\mathrm{Ca}$ to Ni, M4 and M5 have identical abundance ratios, (2) M4 shows overabundances by roughly 0.3 dex for $\mathrm{Si}, \mathrm{Cu}, \mathrm{Zn}$, and all $s$-process elements relative to M5, and (3) for the $r$-process elements, M5 may have slightly higher abundances than M4 by $0.1 \mathrm{dex}$.

We also measure $\mathrm{Mg}$ isotope ratios and find that the ratios are solar in both clusters, with no sign of any star-to-star variation within each cluster. The ratios ${ }^{25} \mathrm{Mg} /{ }^{24} \mathrm{Mg}$ and ${ }^{26} \mathrm{Mg} /{ }^{24} \mathrm{Mg}$ exceed values found in field halo stars at the same metallicity, e.g., Gmb 1830, which implies differences in the clouds from which globular clusters and field halo stars formed. However, we regard these ratios as preliminary, since the spectral resolution was insufficient to accurately distinguish ${ }^{25} \mathrm{Mg}$ from ${ }^{26} \mathrm{Mg}$.

\footnotetext{
3 See GLOBCLUST: Milky Way Globular Clusters Catalog (2003 February version), http://heasarc.gsfc.nasa.gov/W3Browse/star-catalog/globclust.html.
}

There is no clear explanation for the M4 - M5 Si abundance differences since the abundances of Ca should, but do not, follow the behavior of Si. For the elements from Ba to Hf, we find that the mean abundances in M4 and M5 are well explained by scaled versions of the solar $s$ - and $r$-process abundances, albeit with different mixes of $s$ - and $r$-process material for each cluster. Therefore, no new $s$-process site is required to explain the M4-M5 abundance differences for the elements from Ba to Hf. However, although the Th abundances lie above these predictions, the ratio $[\mathrm{Th} / \mathrm{Eu}]$ is identical in both clusters indicating that the universality of the $r$-process extends to Th in these clusters and that no differential decay of Th has occurred, i.e., the clusters have identical ages. The $\mathrm{Pb}$ abundances lie below the predictions, by different amounts for each cluster. Therefore, the sources of the $s$-process may differ between M4 and M5, at least regarding the production of $\mathrm{Pb}$ via the strong component.

The abundance differences between M4 and M5 for $\mathrm{Cu}$ and $\mathrm{Zn}$ are particularly intriguing given that their nucleosynthetic origins continue to be debated. The $s$-process elements, produced in AGB stars (and massive stars), share a similar abundance behavior to $\mathrm{Cu}$ and $\mathrm{Zn}$ in M4 and M5. Updated, but preliminary, yields from AGB models indicate that small amounts of $\mathrm{Zn}$ may be produced only in the most massive AGB stars. These models also predict that the most massive AGB stars may produce $\mathrm{Cu}$ in contrast to our current understanding of $\mathrm{Cu}$ production. Massive AGB stars are expected to produce large amounts of the neutron-rich $\mathrm{Mg}$ isotopes, and observations of high ${ }^{26} \mathrm{Mg} /{ }^{24} \mathrm{Mg}$ ratios in field and cluster stars confirm the AGB yields. However, preliminary measurements show no difference in the $\mathrm{Mg}$ isotope ratios between these two clusters which constrains the contribution of intermediatemass AGB stars to the $\mathrm{Cu}$ and $\mathrm{Zn}$ enhancements in M4. Si, which is produced in massive stars, shows a similar abundance behavior to $\mathrm{Cu}$ and $\mathrm{Zn}$ in M4 and M5. Cu and $\mathrm{Zn}$ may be produced in massive stars via the weak $s$-process. While the abundance ratios $[\mathrm{Rb} / \mathrm{Sr}]$, $[\mathrm{Rb} / \mathrm{Y}]$, and $[\mathrm{Rb} / \mathrm{Zr}]$ are predicted to increase via the weak $s$-process, our measurements do not reveal any cluster-to-cluster variations in these abundance ratios which suggests that either massive stars are not responsible for the $\mathrm{Cu}$ and $\mathrm{Zn}$ differences or that metal-poor massive stars do not alter the $[\mathrm{Rb} / \mathrm{Zr}]$ ratio. Of great interest would be detailed chemical evolution modeling of these two clusters to gain insight into the origin of the $\mathrm{Cu}$ and $\mathrm{Zn}$ abundance differences and therefore their nucleosynthesis production sites.

This research has made use of the SIMBAD database, operated at CDS, Strasbourg, France, and NASA's Astrophysics Data System. D. Y. thanks Inese Ivans and John Norris for valuable discussions and the referee for helpful comments. A. I. K. acknowledges support from the Australian Research Council's Discovery Projects funding scheme (project number DP0664105) and thanks Maria Lugaro for help in developing the extended nucleosynthesis network. D. L. L. thanks the Robert A. Welch Foundation of Houston for support. This research was supported in part by NASA through the American Astronomical Society's Small Research Grant Program.
Abia, C., Busso, M., Gallino, R., Domínguez, I., Straniero, O., \& Isern, J. 2001, ApJ, 559, 1117

Alibés, A., Labay, J., \& Canal, R. 2001, A\&A, 370, 1103

Aoki, W., \& Honda, S. 2008, PASJ, 60/L7

Aoki, W., Honda, S., Sadakane, K., \& Arimoto, N. 2007, PASJ, 59, L15

Asplund, M., Grevesse, N., \& Sauval, A. J. 2005, in ASP Conf. Ser. 336, Cosmic Abundances as Records of Stellar Evolution and Nucleosynthesis, ed. T. G. Barnes III \& F. N. Bash (San Francisco: ASP), 25

Bekki, K., \& Norris, J. E. 2006, ApJ, 637, L109
Belokurov, V., Evans, N. W., Irwin, M. J., Hewett, P. C., \& Wilkinson, M. I. 2006, ApJ, 637, L29

Bernstein, R., Shectman, S. A., Gunnels, S. M., Mochnacki, S., \& Athey, A. E. 2003, in Proc. SPIE, 4841, 1694

Busso, M., Gallino, R., \& Wasserburg, G. J. 1999, ARA\&A, 37, 239

Butler, D., Dickens, R. J., \& Epps, E. 1978, ApJ, 225, 148

Carretta, E., Bragaglia, A., Gratton, R. G., Leone, F., Recio-Blanco, A., \& Lucatello, S. 2006, A\&A, 450, 523

Chieffi, A., \& Limongi, M. 2004, ApJ, 608, 405 
Christlieb, N., et al. 2004, A\&A, 428, 1027

Clayton, D. D., \& Rassbach, M. E. 1967, ApJ, 148, 69

Cohen, J. G., \& Meléndez, J. 2005, AJ, 129, 303

Cottrell, P. L., \& Da Costa, G. S. 1981, ApJ, 245, L79

Cunha, K., Smith, V. V., Suntzeff, N. B., Norris, J. E., Da Costa, G. S., \& Plez, B. 2002, AJ, 124, 379

de Marchi, G., Leibundgut, B., Paresce, F., \& Pulone, L. 1999, A\&A, 343, L9

Den Hartog, E. A., Lawler, J. E., Sneden, C., \& Cowan, J. J. 2003, ApJS, 148, 543

2006, ApJS, 167, 292

Denissenkov, P. A., Da Costa, G. S., Norris, J. E., \& Weiss, A. 1998, A\&A, 333,926

Denissenkov, P. A., \& Herwig, F. 2003, ApJ, 590, L99

Dinescu, D. I., Girard, T. M., \& van Altena, W. F. 1999, AJ, 117, 1792

Fenner, Y., Campbell, S., Karakas, A. I., Lattanzio, J. C., \& Gibson, B. K. 2004, MNRAS, 353, 789

Fenner, Y., Gibson, B. K., Lee, H.-C., Karakas, A. I., Lattanzio, J. C., Chieffi, A., Limongi, M., \& Yong, D. 2003, Publ. Astron. Soc. Australia, 20, 340

Fulbright, J. P. 2000, AJ, 120, 1841

Fulbright, J. P., McWilliam, A., \& Rich, R. M. 2007, ApJ, 661, 1152

García-Hernández, D. A., García-Lario, P., Plez, B., D’Antona, F., Manchado, A., \& Trigo-Rodríguez, J. M. 2006, Science, 314, 1751

Gay, P. L., \& Lambert, D. L. 2000, ApJ, 533, 260

Gratton, R., Sneden, C., \& Carretta, E. 2004, ARA\&A, 42, 385

Gratton, R. G., Sneden, C., Carretta, E., \& Bragaglia, A. 2000, A\&A, 354, 169

Gratton, R. G., \& Ortolani, S. 1988, A\&AS, 73, 137

Gratton, R. G., et al. 2001, A\&A, 369, 87

Grevesse, N., Asplund, M., \& Sauval, A. J. 2007, Space Sci. Rev., 130, 105

Grevesse, N., \& Sauval, A. J. 1998, Space Sci. Rev., 85, 161

Harris, W. E. 1996, AJ, 112, 1487

Heger, A., \& Woosley, S. E. 2002, ApJ, 567, 532

Ivans, I. I., Kraft, R. P., Sneden, C., Smith, G. H., Rich, R. M., \& Shetrone, M. 2001, AJ, 122, 1438

Ivans, I. I., Simmerer, J., Sneden, C., Lawler, J. E., Cowan, J. J., Gallino, R., \& Bisterzo, S. 2006, ApJ, 645, 613

Ivans, I. I., Sneden, C., Kraft, R. P., Suntzeff, N. B., Smith, V. V., Langer, G. E., \& Fulbright, J. P. 1999, AJ, 118, 1273

Käppeler, F., Beer, H., \& Wisshak, K. 1989, Rep. Prog. Phys., 52, 945

Karakas, A. I., Fenner, Y., Sills, A., Campbell, S. W., \& Lattanzio, J. C. 2006a, ApJ, 652, 1240

Karakas, A. I., \& Lattanzio, J. C. 2003, Publ. Astron. Soc. Australia, 20, 279

Karakas, A. I., Lugaro, M., van Raai, M., Sterling, N. C., \& Dinnerstein, H. L. 2008, ApJ, in press (arXiv: 0809.1456)

Karakas, A. I., Lugaro, M. A., Wiescher, M., Görres, J., \& Ugalde, C. 2006b, ApJ, 643, 471

Kurucz, R. 1993, CD-ROM 13, ATLAS9 Stellar Atmosphere Programs and $2 \mathrm{~km} / \mathrm{s}$ Grid (Cambridge: Smithsonian Astrophys. Obs.)

Kurucz, R., \& Bell, B. 1995, CD-ROM 23, Atomic Line Data (Cambridge: Smithsonian Astrophys. Obs.)

Lambert, D. L., Smith, V. V., Busso, M., Gallino, R., \& Straniero, O. 1995, ApJ, 450, 302

Langer, G. E., \& Hoffman, R. D. 1995, PASP, 107, 1177

Lawler, J. E., Den Hartog, E. A., Sneden, C., \& Cowan, J. J. 2006, ApJS, 162, 227

. 2007, ApJS, 169, 120

Lee, Y.-W., Demarque, P., \& Zinn, R. 1994, ApJ, 423, 248

Matteucci, F., Raiteri, C. M., Busson, M., Gallino, R., \& Gratton, R. 1993, A\&A, 272, 421

McWilliam, A., \& Rich, R. M. 1994, ApJS, 91, 749

Meléndez, J., \& Cohen, J. G. 2007, ApJ, 659, L25
Mishenina, T. V., Kovtyukh, V. V., Soubiran, C., Travaglio, C., \& Busso, M. 2002, A\&A, 396, 189

Nilsson, H., Zhang, Z. G., Lundberg, H., Johansson, S., \& Nordström, B. 2002, A\&A, 382, 368

Norris, J. E., \& Da Costa, G. S. 1995, ApJ, 447, 680

Norris, J. E., Da Costa, G. S., \& Tingay, S. J. 1995, ApJS, 99, 637

Odenkirchen, M., et al. 2001, ApJ, 548, L165

Otsuki, K., Honda, S., Aoki, W., Kajino, T., \& Mathews, G. J. 2006, ApJ, 641, L117

Peterson, R. C. 1981, ApJ, 244, 989

Pignatari, M., \& Gallino, R. 2007, Mem. Soc. Astron. Italiana, 78, 543

Primas, F., \& Sobeck, J. 2008, in AIP Conf. Ser. 1001, Evolution and Nucleosynthesis in AGB Stars, ed. R. Guandalini, S. Palmerini, \& M. Busson (Melville, NY: AIP), 230

Pritzl, B. J., Venn, K. A., \& Irwin, M. 2005, AJ, 130, 2140

Prochaska, J. X., Naumov, S. O., Carney, B. W., McWilliam, A., \& Wolfe, A. M. 2000, AJ, 120, 2513

Ramírez, S. V., \& Cohen, J. G. 2002, AJ, 123, 3277

Reddy, B. E., Tomkin, J., Lambert, D. L., \& Allende Prieto, C. 2003, MNRAS, 340,304

Renda, A., et al. 2004, MNRAS, 354, 575

Romano, D., \& Matteucci, F. 2007, MNRAS, 378, L59

Sandage, A., \& Wildey, R. 1967, ApJ, 150, 469

Shetrone, M. D. 1996, AJ, 112, 2639

Simmerer, J., Sneden, C., Cowan, J. J., Collier, J., Woolf, V. M., \& Lawler, J. E. 2004, ApJ, 617, 1091

Simmerer, J., Sneden, C., Ivans, I. I., Kraft, R. P., Shetrone, M. D., \& Smith, V. V. 2003, AJ, 125, 2018

Smith, G. H., Sneden, C., \& Kraft, R. P. 2002, AJ, 123, 1502

Smith, V. V., Suntzeff, N. B., Cunha, K., Gallino, R., Busso, M., Lambert, D. L., \& Straniero, O. 2000, AJ, 119, 1239

Sneden, C. 1973, ApJ, 184, 839

Sneden, C., \& Crocker, D. A. 1988, ApJ, 335, 406

Sneden, C., Gratton, R. G., \& Crocker, D. A. 1991, A\&A, 246, 354

Sneden, C., Johnson, J., Kraft, R. P., Smith, G. H., Cowan, J. J., \& Bolte, M. S. 2000, ApJ, 536, L85

Sneden, C., Kraft, R. P., Shetrone, M. D., Smith, G. H., Langer, G. E., \& Prosser, C. F. 1997, AJ, 114, 1964

Sneden, C., McWilliam, A., Preston, G. W., Cowan, J. J., Burris, D. L., \& Armosky, B. J. 1996, ApJ, 467, 819

Sobeck, J. S., Ivans, I. I., Simmerer, J. A., Sneden, C., Hoeflich, P., Fulbright, J. P., \& Kraft, R. P. 2006, AJ, 131, 2949

Timmes, F. X., Woosley, S. E., \& Weaver, T. A. 1995, ApJS, 98, 617

Tomkin, J., \& Lambert, D. L. 1980, ApJ, 235, 925 1983, ApJ, 273, 722

Travaglio, C., Gallino, R., Arnone, E., Cowan, J., Jordan, F., \& Sneden, C. 2004, ApJ, 601, 864

Travaglio, C., Gallino, R., Busso, M., \& Gratton, R. 2001, ApJ, 549, 346

Umeda, H., \& Nomoto, K. 2002, ApJ, 565, 385

van Raai, M. A., Lugaro, M., Karakas, A. I., \& García-Hernández, D. A. 2008, in AIP Conf. Ser. 1001, Evolution and Nucleosynthesis in AGB Stars, ed. R. Guandalini, S. Palmerini, \& M. Busson (Melville, NY: AIP), 146

Ventura, P., D’Antona, F., Mazzitelli, I., \& Gratton, R. 2001, ApJ, 550, L65 Westin, J., Sneden, C., Gustafsson, B., \& Cowan, J. J. 2000, ApJ, 530, 783

Yong, D., Aoki, W., \& Lambert, D. L. 2006a, ApJ, 638, 1018

Yong, D., Aoki, W., Lambert, D. L., \& Paulson, D. B. 2006b, ApJ, 639, 918

Yong, D., Grundahl, F., Lambert, D. L., Nissen, P. E., \& Shetrone, M. D 2003a, A\&A, 402, 985

Yong, D., Lambert, D. L., \& Ivans, I. I. 2003b, ApJ, 599, 1357

Yong, D., Lambert, D. L., Paulson, D. B., \& Carney, B. W. 2008, ApJ, 673, 854 